\title{
Inhibition of the NFAT Pathway Alleviates Amyloid Beta Neurotoxicity in a Mouse Model of Alzheimer's Disease
}

\author{
Eloise Hudry, ${ }^{1}$ Hai-Yan Wu, ${ }^{1}$ Michal Arbel-Ornath, ${ }^{1}$ Tadafumi Hashimoto, ${ }^{1}$ Roland Matsouaka, ${ }^{2}$ Zhanyun Fan, ${ }^{1}$ \\ Tara L. Spires-Jones, ${ }^{1}$ Rebecca A. Betensky, ${ }^{2}$ Brian J. Bacskai, ${ }^{1}$ and Bradley T. Hyman ${ }^{1}$ \\ ${ }^{1}$ Massachusetts General Hospital, Department of Neurology/Alzheimer's Disease Research Laboratory, Charlestown, Massachusetts 02129, and ${ }^{2}$ Harvard \\ School of Public Health, Boston, Massachusetts 02115
}

Amyloid $\beta(\mathrm{A} \beta)$ peptides, the main pathological species associated with Alzheimer's disease (AD), disturb intracellular calcium homeostasis, which in turn activates the calcium-dependent phosphatase calcineurin (CaN). CaN activation induced by $\mathrm{A} \beta$ leads to pathological morphological changes in neurons, and overexpression of constitutively active calcineurin is sufficient to generate a similar phenotype, even without $\mathrm{A} \beta$. Here, we tested the hypothesis that calcineurin mediates neurodegenerative effects via activation of the nuclear transcription factor of activated T-cells (NFAT). We found that both spine loss and dendritic branching simplification induced by A $\beta$ exposure were mimicked by constitutively active NFAT, and abolished when NFAT activation was blocked using the genetically encoded inhibitor VIVIT. When VIVIT was specifically addressed to the nucleus, identical beneficial effects were observed, thus enforcing the role of NFAT transcriptional activity in A $\beta$-related neurotoxicity. In vivo, when VIVIT or its nuclear counterpart were overexpressed in a transgenic model of Alzheimer's disease via a gene therapy approach, the spine loss and neuritic abnormalities observed in the vicinity of amyloid plaques were blocked. Overall, these results suggest that NFAT/calcineurin transcriptional cascades contribute to A $\beta$ synaptotoxicity, and may provide a new specific set of pathways for neuroprotective strategies.

\section{Introduction}

Amyloid $\beta(\mathrm{A} \beta)$ soluble oligomers are thought to be an important source of neurotoxicity in Alzheimer's disease (AD) (Walsh et al., 2002; Lesné et al., 2006; Shankar et al., 2007; Koffie et al., 2009). While synaptic loss correlates best with cognitive decline (Terry et al., 1991; DeKosky et al., 1996), the molecular mechanisms underlying $\mathrm{A} \beta$ synaptotoxicity and memory impairment remain largely unexplained, and new therapeutic approaches acting to protect neurons toward $A \beta$-related injuries need to be investigated.

Calcium dyshomeostasis and the consequent activation of calcineurin $(\mathrm{CaN}$, or protein phosphatase $2 \mathrm{~B})$ may trigger $\mathrm{A} \beta$ related pathological effects (Liu et al., 2005; Shankar et al., 2007; Kuchibhotla et al., 2008; Wu et al., 2010). An increased CaN activity has been described in AD brains (Liu et al., 2005; Wu et al., 2010), and $\mathrm{CaN}$ activation was reported in astrocytes sur-

Received Dec. 23, 2011; accepted Jan. 12, 2012.

Author contributions: E.H., H.-Y.W., T.L.S.-J., and B.T.H. designed research; E.H. performed research; H.-Y.W. M.A.-O., T.H., Z.F., T.L.S.-J., and B.J.B. contributed unpublished reagents/analytic tools; E.H., R.M., and R.A.B. analyzed data; E.H. and B.T.H. wrote the paper.

This work was supported by National Institutes of Health Grants AG08487, EB000768, and EY13399, the Lefler fellowship (Harvard Medical School), and the French Bettencourt-Schueller award for young scientists. We thank Dr. Norris (Sanders-Brown Center on Aging, Lexington, KY) for providing the VIVIT-GFP constructs and Dr. Ruthazer (Montreal Neurological Institute, McGill University, Montreal, QC, Canada) for providing the NLS-VIVIT-GFP plasmid. We thank Dr. Alberto Serrano-Pozo for helpful comments during manuscript preparation and Daniel Joyner for primary neuron preparation.

The authors declare no competing financial interests.

Correspondence should be addressed to Dr. Bradley T. Hyman, Massachusetts General Institute for Neurodegenerative Disease, 114 16th Street, Charlestown, MA 02129. E-mail: bhyman@partners.org.

DOI:10.1523/JNEUROSCI.6439-11.2012

Copyright $\odot 2012$ the authors $\quad 0270-6474 / 12 / 323176-17 \$ 15.00 / 0$ rounding amyloid deposits, thus exacerbating neuroinflammation (Norris et al., 2005). In neurons, CaN activation leads to pathological morphological changes, which could be blocked by abolition of CaN enzymatic activity using FK506 (Wu et al., 2010; Rozkalne et al., 2011). FK506 also improves cognitive deficits in the Tg2576 AD mouse model (Taglialatela et al., 2009), thus making calcineurin a potential therapeutic target. However, because FK506 prevents CaN phosphatase activity in all the cell types and toward all its substrates, the mechanisms underlying FK506 neuroprotection are unclear.

Calcineurin is a unique neuronal $\mathrm{Ca}^{2+} /$ Calmodulin-dependent serine/threonine phosphatase that plays fundamental physiological roles during development and regulates processes such as neurotransmitter release, synaptic plasticity, and learning (Klee et al., 1979; Groth et al., 2003). Upon activation, CaN leads to post-translational modification of postsynaptic proteins such as cofilin (Zhou et al., 2004) and AKAP79, which is associated with long-term depression (Bhattacharyya et al., 2009; Jurado et al., 2010). In addition, CaN dephosphorylates the nuclear factor of activated T cells (NFAT), which induces its nuclear translocation and the expression of target genes implicated in neuronal survival, axonal outgrowth, and dendritic complexity (Benedito et al., 2005; Nguyen and Di Giovanni, 2008; Schwartz et al., 2009). $\mathrm{CaN}$ activation therefore triggers short-term effects directly at the synapse as well as long-term modifications of synaptic plasticity by modulating gene expression through NFAT.

To decipher more precisely the role of CaN activation in Alzheimer disease, we tested the hypothesis that neuronal morphological changes caused by $\mathrm{A} \beta$ may be improved by inhibiting the CaN-induced NFAT pathway. We used a genetically encoded 
VIVIT peptide initially developed as a specific competitive inhibitor of the interaction between $\mathrm{CaN}$ and NFAT, without affecting CaN phosphatase activity (Aramburu et al., 1999). We established that (1) VIVIT efficiently inhibits the neurotoxic effects associated with a constitutively activated $\mathrm{CaN}$ (CACaN); (2) expression of a constitutively active form of NFAT produces a phenocopy of the effects induced by $\mathrm{CACaN}$ or by $\mathrm{A} \beta$ exposure; (3) $\mathrm{A} \beta$-induced decrease of spine density and dendritic complexity is inhibited by VIVIT; (4) restricted expression of VIVIT in the nucleus retains the beneficial effects of VIVIT, whereas a membrane-bound version of this peptide has no effect; (5) changes associated with amyloid plaques are alleviated when VIVIT or NLS-VIVIT were introduced by AAV-mediated gene delivery in the cortex of an $\mathrm{AD}$ mouse model in vivo.

Together, our findings offer new therapeutic opportunities by targeting $\mathrm{A} \beta$ downstream events through inhibition of NFAT transcriptional pathways.

\section{Materials and Methods}

Culture of primary cortical neurons and collection of neuronal conditioned media. Primary neurons were cultured from cerebral cortices of embryonic day 16 mice (Charles River Laboratories). Embryos resulted from the mating between a $T g 2576$ male that heterozygously overexpresses a human mutated APP gene and a wild-type female, thus giving rise to both transgenic ( $\mathrm{Tg}$ ) and littermate (Wt) cultures. The genotype of each embryo was determined by PCR. Neurons were prepared as previously described (Wu et al., 2010) and plated to a density of $6.7 \times 10^{5}$ viable cells $/ 35 \mathrm{~mm}$ culture dishes coated with poly-D-lysine $(100 \mu \mathrm{g} / \mathrm{ml}$, SigmaAldrich). The medium of the cells was not renewed to allow $\mathrm{A} \beta$ peptides to accumulate and conditioned media were collected from 14 DIV cultures (Wu et al., 2010). The concentration of $A \beta$ peptides was quantified by a mouse/human ELISA kit (Wako) and reached $\sim 7000 \mathrm{pMol}$. Transfection experiments were done at 5 DIV using Lipofectamine 2000 (Invitrogen).

Spine density and Sholl analyses. At 5 DIV, primary neurons were transfected with either pEGFP-N1 or pDsRed-Express-N1 plasmids (Clontech Laboratories), altogether with pVIVIT-EGFP and pVIVIT-NLS-EGFP constructs generously provided by Dr. Norris (Sanders-Brown Center On Aging, University of Kentucky, Lexington, KY) and Dr. Ruthazer (McGill University, Montreal, QC, Canada), respectively. For a subset of experiments, cells were also transfected with a wild-type or a constitutively active form of calcineurin described previously (Wu et al., 2010). Live imaging of GFP- or DsRed-expressing primary neurons (18 DIV) was used to analyze the morphological parameters of the cells. Images of the whole cell and of dendritic segments were captured using a LSM 510 Zeiss microscope to determine the neuritic complexity and the spine density. GFP and DsRed were respectively excited at 488 and $543 \mathrm{~nm}$ and emitted light was collected between $500-550 \mathrm{~nm}$ and 565-615 nm. Dendritic spines were analyzed using the NeuronStudio software (CNIC tools) that automatically detects three different spine types (thin, stubby, and mushroom) according to their morphological measures (i.e., length of the "neck" and size of the "head" of each spine) (Rodriguez et al., 2008). The complexity of the neuronal dendritic tree was determined by Sholl analysis (Spires-Jones et al., 2011), reporting the number of branch points with respect to the distance from the cell body. All these parameters were evaluated on transfected cells that did not show any obvious morphological alterations such as dendritic dystrophies.

Immunocytochemistry. Immunostaining was performed using a standard protocol (Wu et al., 2010). Briefly, primary neurons were fixed with $4 \%$ paraformaldehyde in PBS, pH 7.4, for 15 min, permeabilized with $0.5 \%$ Triton X-100 in PBS for 20 min, and blocked with 3\% bovine serum albumin at room temperature for $1 \mathrm{~h}$. Primary antibodies to detect NFATc4 (polyclonal s.c.-13036, 1:200 dilution, Santa Cruz Biotechnology), laminin-B1 (polyclonal ab16048, 1:1000, Abcam), HA tag (Clone 16B12, 1:200 dilution, Covance), and GFP (polyclonal ab6556, 1:1000, Abcam or chicken anti-GFP, Aves) were incubated overnight at $4^{\circ} \mathrm{C}$. Secondary antibodies conjugated to either cyanine 3 (Cy3, 1:1000; Jack- son ImmunoResearch), cyanine 5 (Cy3, 1:500; Jackson ImmunoResearch), or Alexa 488 (1:1000; Invitrogen, Eugene, OR) were then applied. Fluorescent images were obtained using a LSM 510 Zeiss confocal microscope.

Evaluation of NFAT subcellular localization. The changes in the subcellular distribution of NFATc 4 were evaluated after immunostaining of the endogenous protein in primary neurons or in mouse brain slices. GFPpositive neurons that were previously transfected or transduced with GFP, VIVIT-GFP, or NLS-VIVIT-GFP were specifically analyzed. Using ImageJ (National Institutes of Health open software), the fluorescence intensity of NFATc4 in the nucleus was determined by overlap with the nuclear staining DAPI (Vector Laboratories), whereas the fluorescence intensity in the cytoplasm was quantified in the rest of the cell body, excluding the nuclear compartment (Fig. $1 C$ ). The intensity of nuclear NFATc4 was then divided by the intensity of cytoplasmic NFATc4.

Evaluation of NFAT transcriptional activity using a Luciferase reporter system. Cortical primary neurons (7 DIV) were transduced with an AAVNFAT-TA-Luc, altogether with AAV-wtCaN or AAV-CACaN and AAV-GFP, AAV-VIVIT-GFP, or AAV-NLS-VIVIT-GFP. Three days after transduction, cells were harvested and the luciferase activity was measured with a luminometer using a reagent kit (Luciferase Assay System with Reporter Lysis Buffer; Promega). The background luciferase activity, calculated when pNFAT-Luc alone was added, was subtracted from all experiments.

Animals. In vivo experiments were performed using APPswe/PS1d9 double transgenic mice (APP/PS1, obtained from Jackson Laboratory) that overexpress a human mutant amyloid precursor protein gene containing the Swedish mutation K594N/M595L and a variant of the Presenilin 1 gene deleted for the exon 9, both under the control of PrP promoter (Jankowsky et al., 2004). Substantial amyloid deposition is visible by 6 months of age and we used 7-month-old animals. Wild-type littermates were used as controls. Experiments were performed in accordance with NIH and institutional guidelines.

Viral vectors construction and production. Plasmids containing VIVITGFP and NLS-VIVIT-GFP were digested using NotI and NcoI restriction enzymes and subcloned into an AAV backbone containing the chicken $\beta$-actin promoter and a Woodchuck Hepatitis Virus PostTranscriptional Regulatory Element (WPRE). The Myr-tagged VIVITGFP was obtained after amplification by PCR and insertion into an AAV backbone using Xho1 and BsrG1 restriction sites. The AAV-NFAT-TALuc plasmid was purchased from Clontech Laboratories and the constructions of AAV-CBA-HA-wtCaN and pAAV-CBA-HA-CACaN backbones were described previously (Wu et al., 2010). As a control, we used an AAV-GFP that was available at the Harvard Gene Therapy Core. High titer of AAV serotype 2 vectors (AAV2-GFP, AAV2-VIVIT-GFP and AAV2-NLS-VIVIT-GFP, AAV2-WtCaN, AAV2-CACaN and AAV2NFAT-TA-Luc) were produced after triple-transfection of HEK293 cells by the Harvard Gene Therapy Core, except for the AAV8-tdTomato that was provided by the Gene Transfer Vector Core from University of Iowa, Iowa City, IA. Lentiviral vectors coding for WtNFAT and CANFAT were obtained after amplification of HA-mNFATc4 (WtNFAT) and HA-CAmNFATc4 (CANFAT) by PCR. Both PCR products were then digested by NheI and BsrGI restriction enzymes and cloned into a pCSCW2-IRES lentiviral backbone. Lentiviral vectors were produced by the Vector Development and Production Core at Massachusetts General Hospital. All the constructs were verified by sequencing.

Stereotactic intracortical injections. Stereotactic intracortical injections of AAV serotype 2 vectors were performed as described previously (Spires et al., 2005). Animals were anesthetized by intraperitoneal injection of ketamine/ xylazine (100 and $50 \mathrm{mg} / \mathrm{kg}$ body weight, respectively) and positioned on a stereotactic frame (David Kopf Instruments). Injections of vectors were performed in the cerebral cortex of each hemisphere with $3 \mu$ l of viral preparation (titer $2 \times 10^{12} \mathrm{vg} / \mathrm{ml}$ ) using a 33-gauge sharp micropipette attached to a $10 \mu \mathrm{l}$ Hamilton syringe (Hamilton Medical) at a rate of $0.12 \mu \mathrm{l} / \mathrm{min}$. Stereotactic coordinates of injection sites were calculated from bregma (anteroposterior $-1 \mathrm{~mm}$, mediolateral $\pm 1 \mathrm{~mm}$, and dorsoventral $-1.2 \mathrm{~mm}$ ).

Cranial window implantation and multiphoton imaging. Four weeks after AAV intracortical injection, mice were anesthetized with isoflurane $(1.5 \%)$ and a cranial window was implanted by replacing a piece of skull 
A
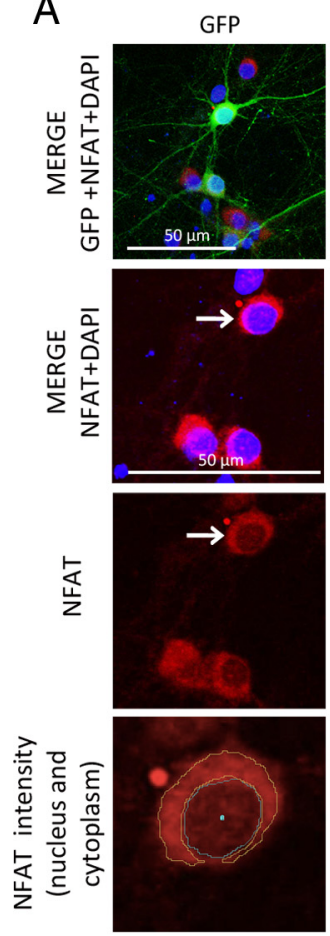

D
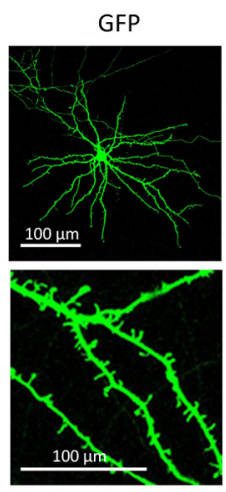

WtCaN+GFP
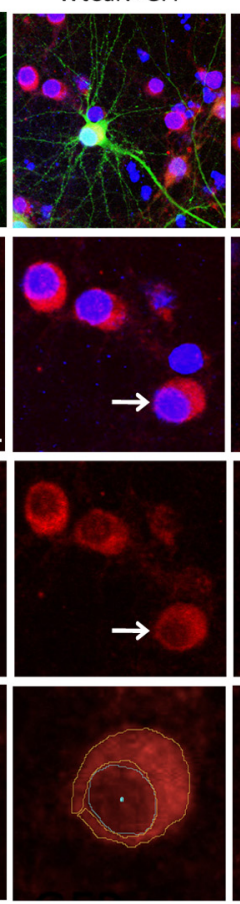

$\mathrm{WtCaN}+\mathrm{GFP}$
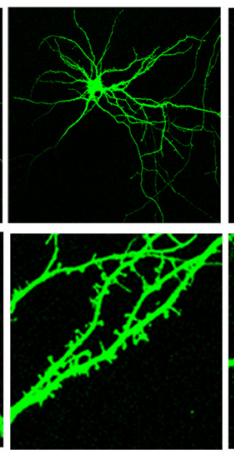
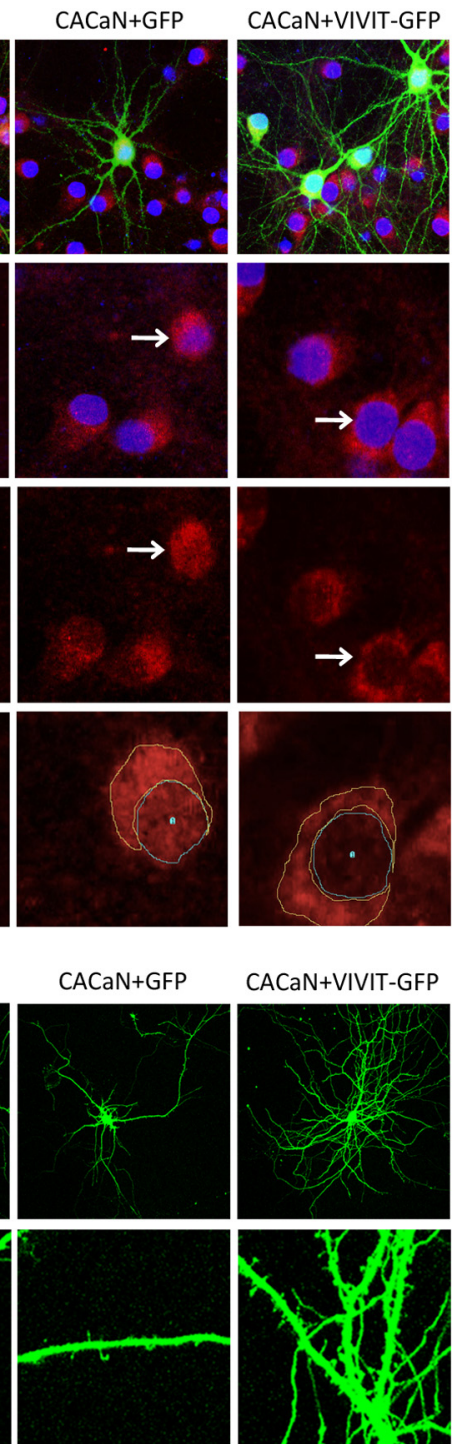
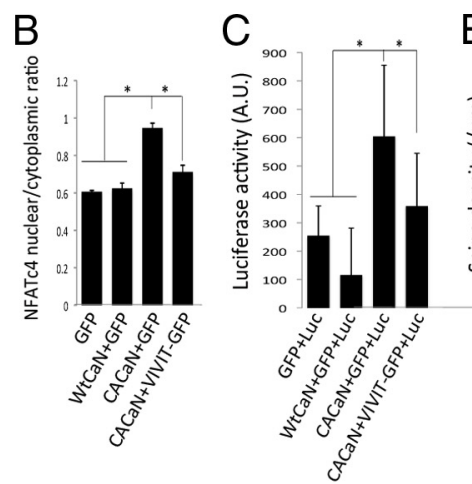

$E$

$\mathrm{F}$
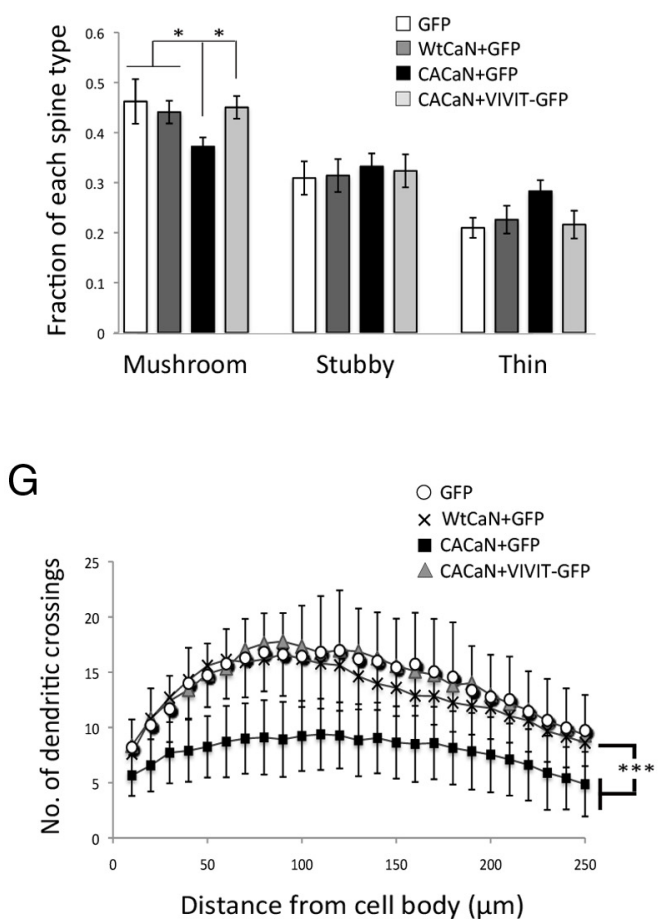

Figure 1. VIVIT-GFP overexpression counteracts the effects of constitutively activated calcineurin. $\boldsymbol{A}$, Representative pictures of GFP, NFAT c4 immunostaining, and DAPI (top row) in cortical primary neurons transfected with WtCaN or CACaN, altogether with either GFP or VIVIT-GFP (transfected cells are indicated by an arrow). An increased colocalization of the NFATc4 immunostaining and DAPI is detected in cells overexpressing CACaN and GFP (second row), which is abolished when VIVIT-GFP is cotransfected. The bottom row represents the detection of NFATc4 immunostaining in the nucleus and in the cytoplasm using ImageJ.Scale bar, $50 \mu \mathrm{m}$. B, NFATc4 ratios between the nucleus and the cytoplasm are significantly increased when CACaN is overexpressed compared with GFP and WtCaN + GFP. When CACaN is co-transfected with VIVIT-GFP, the nucleus/cytoplasm ratios are significantly decreased and become comparable to neurons transfected with WtCaN + GFP. C, Analysis of NFAT transcriptional activity using the Luciferase reporter system shows that overexpression of CACaN leads to a significant higher Luciferase signal (i.e., higher NFAT transcriptional activity) compared with WtCaN and CACaN + VIVIT. D, E, Representative images and spine density quantification of neurons transfected with GFP, WtCaN/GFP, CACaN/GFP, or CACaN/VIVIT-GFP show that cells co-transfected with CACaN and GFP have a decreased spine density compared with GFP or WtCaN + GFP overexpressing neurons, which is significantly increased by VIVIT-GFP. Scale bar, $100 \mu \mathrm{m}$. F, The proportion of each spine type is also affected by CACaN and a significant decrease of the more mature "mushroom" spines is observed compared with GFP and WtCaN/GFP transfected neurons. This effect is greatly abolished when VIVIT-GFP is overexpressed. G, The overall dendritic complexity is also improved when the VIVIT inhibitor was coexpressed with CACaN compared with CACaN/GFP transfected cells. No statistical difference is observed between GFP, WtCaN/GFP, and CACaN/VIVITGFP transfected cells. ${ }^{*} p<0.05{ }^{* *} p<0.001$ and ${ }^{* * *} p<10^{-5}$. ( $n>40$ cells per condition).

by a glass coverslip of $8 \mathrm{~mm}$ diameter [as described previously (Spires et al., 2005)]. For imaging, a wax ring was built on the edge of the window to create a well of water for the objective (Olympus XLPlan N 25X objective with a numerical aperture of 1.05). To visualize the amyloid deposits, transgenic animals received an intraperitoneal injection of methoxy- $\mathrm{XO}_{4}(5 \mathrm{mg} / \mathrm{kg}) 24 \mathrm{~h}$ before surgery, a fluorescent compound that crosses the blood-brain barrier and binds to amyloid deposits (Bacskai et al., 2002; Klunk et al., 2002). When needed, Texas Red dextran (70,000 Da molecular weight; $12.5 \mathrm{mg} / \mathrm{ml}$ in sterile PBS; Invitrogen) was injected into a lateral tail vein to provide a fluorescent angiogram.

GFP-filled neuronal processes, amyloid deposits (in the case of APP/PS1 animals), and blood vessels were imaged using the Olympus FluoView FV1000MPE multiphoton laser-scanning system mounted on an Olympus BX61WI microscope (Olympus). A DeepSee Mai Tai Ti:sapphire mode-locked laser (Mai Tai; Spectra-Physics) generated two-photon fluorescence with $860 \mathrm{~nm}$ excitation. Emitted light was detected through three filters in the range of 420-460, 495-540, and $575-630 \mathrm{~nm}$ (Hamamatsu). Neurites were imaged at depth of 50-200 $\mu \mathrm{m}$ from the surface of the brain. High-resolution images were captured using the optical zoom feature in the Fluoview software $(63 \times$ $63 \times 1 \mu \mathrm{m}$ sections; $40-100$ sections per stack).

Image processing and analysis. Two-dimensional projections of GFPfilled neurites were obtained using ImageJ. Only dendrites that were at least $20 \mu \mathrm{m}$ long and had prominent dendritic spine protrusions were considered for analysis. Spine density and morphology were evaluated using the NeuronStudio software, as described previously (Rodriguez et 
al., 2008). Distances to plaques were evaluated by the average of three different measures between each neurite (two measures at each endpoint and one measure at the midpoint) and the edge of the nearest amyloid deposit.

Immunohistological analysis. One month after implantation of the cranial window, mice were killed by $\mathrm{CO}_{2}$ inhalation. One cerebral hemisphere was fixed in 4\% paraformaldehyde in PBS whereas the other hemisphere was snap frozen in liquid nitrogen. To detect GFP and NFATc4, paraffin-embedded sections $(10 \mu \mathrm{m})$ were sequentially deparaffinized in xylene, rehydrated in ethanol, treated in citrate buffer $(10 \mathrm{~mm}$ Sodium Citrate, 0.05\% Tween 20, pH 6.0), permeabilized in PBS with $0.5 \%$ Triton, blocked in PBS with 3\% BSA, and incubated overnight with the primary antibody. Incubation with the secondary antibody was done for $2 \mathrm{~h}$ at room temperature the next day.

Statistical analysis. Except for the Sholl's plot analyses, statistical analyses were done using Statview and JMP softwares. In vitro experiments were done at least in triplicate. In vivo, at least five animals were included per condition. Equality of variances ( $F$ test) was verified between each date of experiment and between animals of the same group. We made comparisons between groups by one- or two-way ANOVA followed by post hoc Bonferroni correction for multiple comparisons among means. Data are presented as mean \pm SD.

Sholl's plot analyses were represented as the mean of the number of intersections according to the distance from the cell body, and statistics were done using a newly developed analytical approach [using R software (Spires-Jones et al., 2011)]. To assess the difference in length and complexity of dendritic arborization between each treatment, the area under the curve (AUC) was estimated through a trapezoidal method, which reflects the overall complexity of the dendritic arborization. A Wilcoxon rank sum test followed by a Bonferroni $p$ value adjustment procedure was applied. For all the analyses, differences with a $p$ value of $<0.05$ were considered statistically significant.

\section{Results}

Genetically encoded VIVIT blocks NFAT accumulation in the nucleus and prevents spine loss and dendritic simplification induced by constitutively active calcineurin (CaN)

The use of pharmacological inhibitors of $\mathrm{CaN}$ phosphatase activity, such as FK506, is associated with a broad range of side-effects in transplanted patients (Ponticelli and Campise, 2005; Lee et al., 2008). In an effort to develop a safer drug, Aramburu et al. (1999) screened for an NFAT-specific reagent and developed a new inhibitor peptide called VIVIT (16 aa, MAGPHPVIVITGPHEE) that was able to interact with the CaN docking motif of NFAT with a high affinity, without interfering with its enzymatic activity.

In vitro, we first tested whether the overexpression of VIVITGFP in cortical primary neurons was able to counteract the nuclear translocation and the transcriptional activity of NFATc4 [the most prominent NFAT isoform in neurons (Ho et al., 1994)], as well as the neuronal morphological changes induced by a constitutively active form of calcineurin (CACaN). The constitutively active CaN was initially identified as a calpaindependent truncated product of calcineurin $(45 \mathrm{kDa})$, in which the regulatory autoinhibitory domain was removed, so that CACaN has an enhanced phosphatase activity (Wu et al., 2004). By contrast, overexpression of wild-type calcineurin (WtCaN) is tightly regulated and does not lead to an increase of calcineurin activity. At 5 DIV, cortical primary neurons were cotransfected with either CACaN or WtCaN and with either GFP or VIVITGFP. Cells were cultured for 16-18 DIV before analysis. Because of the presence of an HA-tag in the WtCaN and CACaN constructs, we verified that $>90 \%$ of GFP-filled neurons also coexpressed each form of $\mathrm{CaN}$ (data not shown). As expected, the nuclear/cytoplasmic ratio of NFATc4 immunofluorescence intensity was significantly higher when CACaN was overexpressed $(0.94 \pm 0.025)$ compared with WtCaN $(0.62 \pm 0.028)$, whereas
WtCaN by itself did not affect NFAT subcellular localization compared with GFP transfected cells $(0.67 \pm 0.08)$ (Fig. $1 A, B)$. This suggests that increased CaN activity leads to an accumulation of NFATc4 in the nucleus. By contrast, co-transfection of CACaN with VIVIT-GFP prevents the nuclear accumulation of NFATc4, as indicated by lower nuclear/cytoplasmic ratios $(0.62 \pm 0.036)$ that were comparable to WtCaN transfected neurons (Fig. $1 A, B$ ). When VIVIT-GFP alone was overexpressed, no change could be detected in the subcellular localization of NFATc4 and the nuclear/cytoplasmic ratio remained comparable to GFP-transfected cells (data not shown). To further evaluate the effect of VIVIT on NFAT transcriptional activity, a reporter system containing several NFAT transcriptional response elements driving the expression of luciferase was used. Primary neurons were transduced with AAV-GFP, AAV-WtCaN+GFP, AAV-CACaN+AAV-GFP, AAV-CACaN+AAV-VIVIT-GFP at $10 \mathrm{DIV}$, and luciferase activity was quantified after $3 \mathrm{~d}$. Overexpression of a constitutively active form of calcineurin led to an increase of NFAT transcriptional activity compared with $\mathrm{WtCaN}$. When VIVIT-GFP was co-transduced with CACaN, a significant decrease of the Luciferase activity was observed, suggesting that VIVIT efficiently inhibits NFAT-dependent transcriptional activity (Fig. 1C).

To determine whether VIVIT-GFP was able to prevent the pathological morphological changes induced by $\mathrm{CACaN}$, we analyzed the spine density and dendritic complexity of GFP or VIVIT-GFP-expressing neurons that were also transfected with $\mathrm{WtCaN}$ or CACaN plasmids. The density of spines in CACaN/ GFP overexpressing neurons $(0.18 \pm 0.046$ spines $/ \mu \mathrm{m})$ was significantly lower compared with cells overexpressing WtCaN/GFP $(0.42 \pm 0.09$ spines/ $\mu \mathrm{m}$; Fig. $1 D, E)$. Sholl's plot analyses also indicated that uncontrolled activation of calcineurin in neurons induced a marked dendritic simplification compared with wildtype calcineurin (Fig. $1 D, G$ ). However, when VIVIT-GFP was introduced together with $\mathrm{CACaN}$, a significantly higher spine density $(0.4 \pm 0.081$ spines $/ \mu \mathrm{m})$ and a more complex neuritic arborization were observed, so that these morphological parameters reached those of WtCaN transfected neurons (Fig. 1D,E, $G)$. Importantly, no difference was observed between cells that were transfected with GFP alone $(0.43 \pm 0.13$ spines $/ \mu \mathrm{m})$ or $\mathrm{WtCaN}+\mathrm{GFP}(0.42 \pm 0.09$ spines/ $\mu \mathrm{m})$, suggesting that WtCaN did not change the spine density and dendritic complexity of cortical neurons in culture (Fig. $1 D, E, G$ ). Similarly, overexpression of VIVIT-GFP alone did not affect the morphological parameters of the cells (data not shown). The beneficial effect of VIVIT-GFP was therefore specifically related to the inhibition of CACaN-dependent NFAT activation.

Interestingly, when the different spine types were compared, a decreased proportion of "mushroom" type spines was associated with CACaN+GFP $(38 \% \pm 1.8 \%)$ compared with GFP $(47 \% \pm$ $2.8 \%)$, WTCaN + GFP $(44 \% \pm 2.3 \%)$, or CACaN + VIVIT-GFP $(46 \% \pm 1.4 \%)$ (Fig. $1 F)$, implicating calcineurin activation in the collapse of mature, mushroom-shaped spines that are known to be more stable than thin spines (Holtmaat et al., 2005).

\section{Constitutively active NFAT induces morphological abnormalities in primary neurons that phenocopy the effects of constitutively active $\mathrm{CaN}$}

As both spine loss and dendritic simplification were rescued when a genetically encoded VIVIT-GFP was overexpressed in neurons, we postulated that the morphological effects of CACaN were related to NFAT activation. We therefore investigated the specific effects of NFATc4 on these morphological parameters. We transduced 14 DIV cortical neurons with ei- 
A
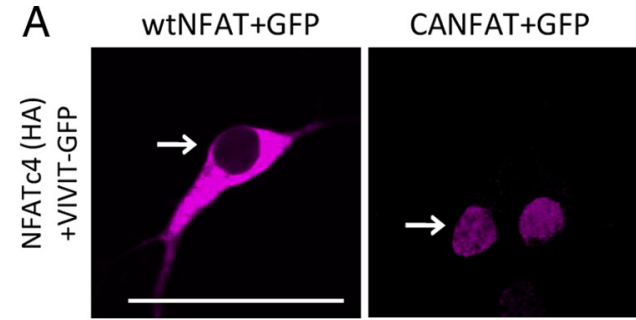

CANFAT+VIVIT-GFP
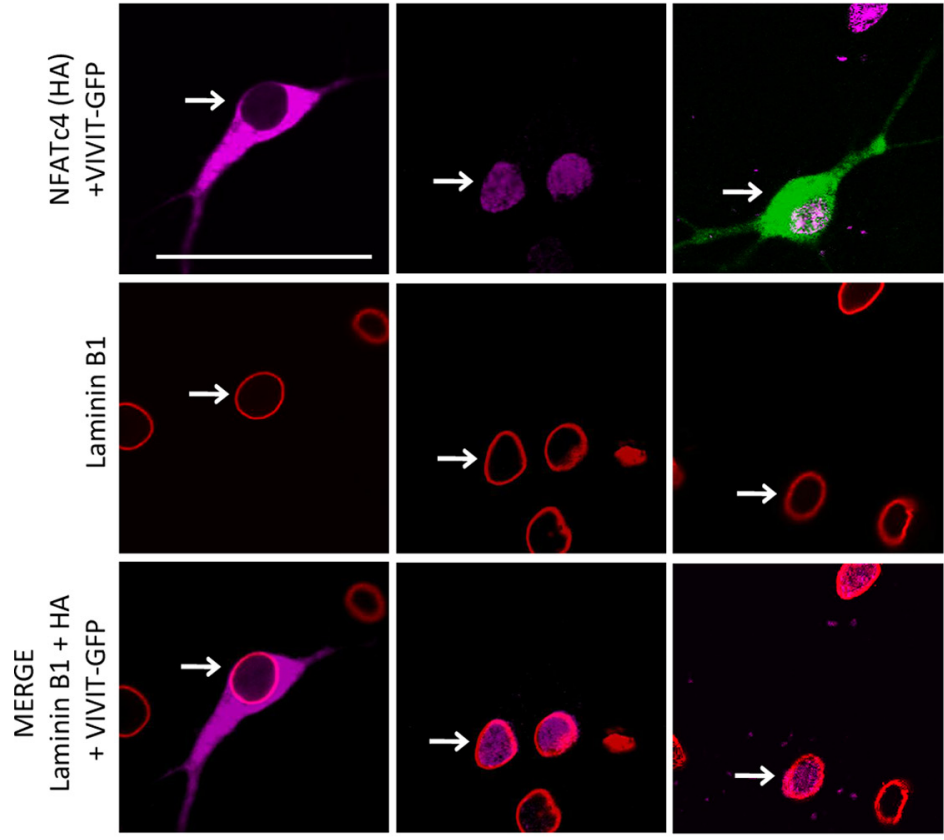

B
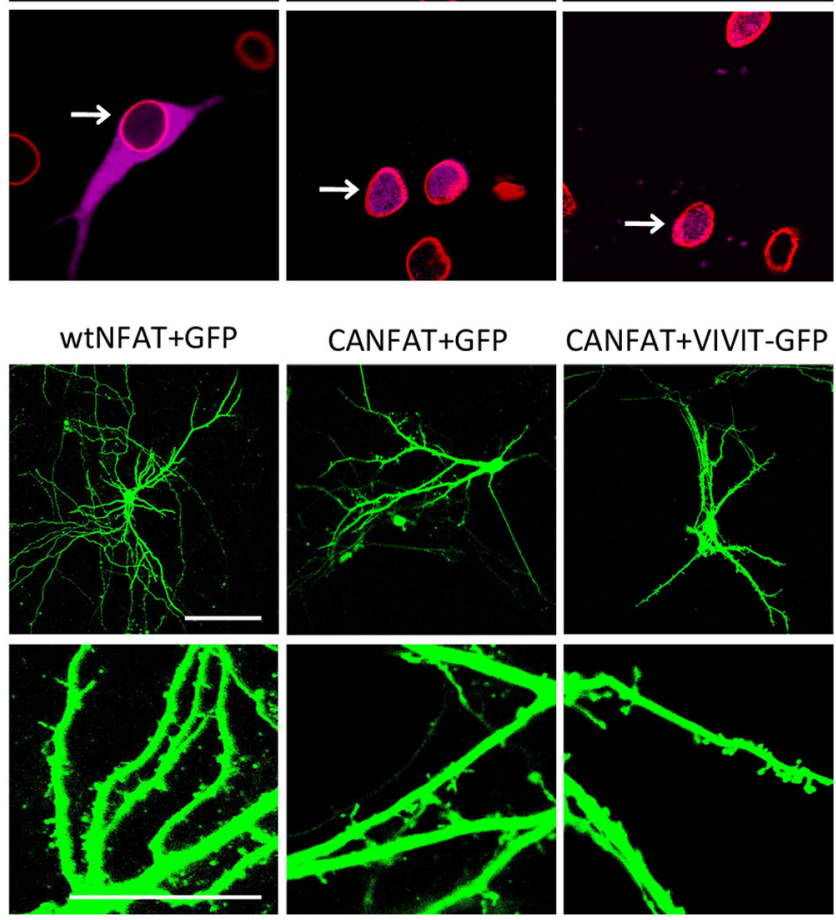

C
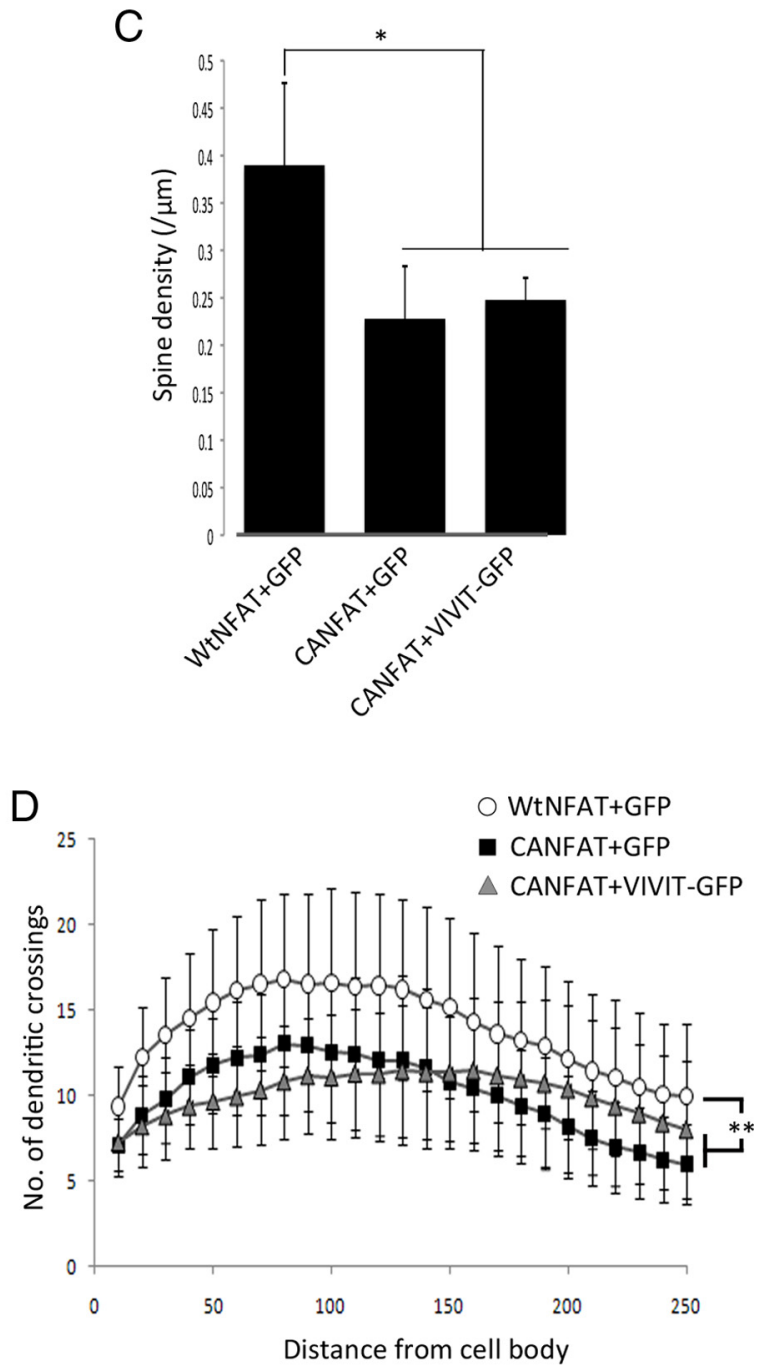

Figure 2. Overexpression of a constitutively activated NFATc4 is sufficient to induce abnormal morphological changes in primary neurons. $\boldsymbol{A}$, Representative images of the nuclear membrane marker laminin B1 and of HA-tagged NFATc4 in cortical neurons that were transduced with WtNFAT/CANFAT (lentiviral vectors). WtNFAT (left) is mainly present in the cytoplasm, outside the nucleus delimited by laminin B1. On the contrary, the constitutively activated form of NFATC4 was concentrated into the nucleus of the cells and fit the laminin B1 staining. The nuclear localization of CANFAT is not affected by the overexpression of VIVIT-GFP (right). Scale bar, $10 \mu \mathrm{m}$. B, Images of GFP-filled neurons (top) and dendrites (bottom) that were transduced with either WtNFAT or CANFAT. Scale bar, $100 \mu \mathrm{m}$. C, Overexpression of CANFAT is associated with a decreased number of spines per dendritic segment compared with WtNFAT transduced cells. The loss of dendritic spines observed in CANFAT-expressing neurons is not improved by VIVIT-GFP. D, The overall dendritic complexity is also decreased by CANFAT compared with WtNFAT. The lower dendritic complexity induced by CANFAT cannot be rescued by VIVIT-GFP ( $p>0.18$ when compared with CANFAT/GFP transfected cells). ${ }^{*} p<0.05$ and ${ }^{* *} p<0.01$. ( $n>30$ cells per condition).

ther a lentiviral vector encoding a HA-tagged wild-type (WtNFAT) or constitutively activated (CANFAT) form of NFATc4 that lacks its $\mathrm{N}$ terminus regulatory CaN binding domain. This truncated NFATc4 is therefore activated without calcineurin (Molkentin et al., 1998). To detect morphological variables, neurons were previously transfected with GFP at 5 DIV and we verified that a high percentage $(80 \%)$ of the GFP-filled cells were also positive for HA. As observed in Figure $2 A$, WtNFAT was mainly detected in the cytoplasm and was excluded from the nucleus (delimited by the marker of the nuclear membrane laminin-B1), whereas, as expected, the constitutively active form of NFATc4 was concentrated in the nucleus. Importantly, CaN activity was not previously induced in these cells (either by using a calcium ionophore or CACaN overexpression), demonstrating that CANFAT did not depend upon any other upstream events to be activated. In contrast to CACaN-induced NFATc4 nuclear recruitment, accumulation of CANFAT into the nucleus could not be prevented by VIVIT-GFP (Fig. $2 A$ ). Overexpression of CANFAT led to a significant decrease of spine density $(0.22 \pm 0.045$ spines $/ \mu \mathrm{m})$ and dendritic simplification compared with $\mathrm{Wt}$ NFAT $(0.39 \pm 0.07$ spines $/ \mu \mathrm{m})$. None of these changes could be improved by co-transfection with VIVIT-GFP (0.24 \pm 0.023 spines $/ \mu \mathrm{m}$ ) (Fig. $2 B-D$ ), thus demonstrating that VIVIT acts upstream of NFAT activation and does not have an effect nonrelated to calcineurin. We therefore concluded that ectopic expression of a constitutively active form of NFATc4 is sufficient to induce spine loss and dendritic simplification in primary neurons, suggesting that NFATc4-related transcriptional events are critical for $\mathrm{CaN}$-induced neurotoxicity in vitro. 


\section{Overexpression of VIVIT in Tg2576 transgenic neurons or in wild-type neurons treated with $\mathrm{A} \boldsymbol{\beta}$-containing medium rescues their morphological abnormalities}

We previously observed that both transgenic neurons cultured from $\operatorname{Tg} 2576$ embryos and wild-type neurons treated with $\mathrm{A} \beta$ containing conditioned media develop AD-like pathological morphological changes that include dendritic spine loss and neuritic simplification. The involvement of $\mathrm{CaN}$ in these processes was suggested by the fact that both phenotypes could be rescued when FK506 was added to the medium or used in transgenic mice (Wu et al., 2010; Rozkalne et al., 2011). Here we showed that the overexpression of constitutively activated CaN or NFAT phenocopy the morphological changes induced by $\mathrm{A} \beta$. Based on these observations, we asked whether the inhibition of NFAT activation by calcineurin can also alleviate $\mathrm{A} \beta$ synaptotoxicity.

We first tested the efficiency of VIVIT-GFP in transgenic neurons that were cultured from Tg2576 embryos. Because the mutated $A P P$ transgene was present as a heterozygous state, transgenic ( $\mathrm{Tg}$ ) or littermate $(\mathrm{Wt})$ cultures could be analyzed in parallel. Cells were transfected with either GFP or VIVITGFP at 5 DIV and analyzed at 18 DIV. A decreased density of dendritic spines was observed in Tg neurons $(0.21 \pm 0.11$ spines $/ \mu \mathrm{m})$ compared with their wild-type counterpart $(0.45 \pm 0.1$ spines $/ \mu \mathrm{m})$ (Fig. $3 A, B)$. The spine loss detected in $\mathrm{Tg}$ neurons was also associated with a simplification of the dendritic tree (Fig. 3C). Importantly, when VIVIT-GFP was overexpressed in Tg neurons, both parameters were rescued to normal wild-type levels (spine density: $0.39 \pm 0.12$ spines/ $\mu \mathrm{m})$ and significantly improved compared with Tg neurons transfected with GFP alone.

To study the effect of exogenous $\mathrm{A} \beta$ peptides on neuronal morphological parameters, $\mathrm{A} \beta$-containing conditioned medium (TgCM) was obtained by collecting the medium of Tg neurons after 2 weeks in culture and used to treat naive wild-type cells. The concentration of $\mathrm{A} \beta_{40}$ was estimated as $7000 \mathrm{pM}$ and was comparable to the levels observed in AD brains (Ingelsson et al., 2004). Western blot analysis showed that TgCM mainly contained soluble oligomeric species (data not shown) that have been described to have important neurotoxic effects (Walsh et al., 2002; Lesné et al., 2006; Shankar et al., 2007, 2008). Wild-type primary neurons cultured in standard NB/B27-serum free medium for 16 DIV were exposed for $24 \mathrm{~h}$ to either wild-type (WtCM) or transgenic (TgCM) conditioned media diluted 1:1 in the initial medium. As observed in Figure 3, $D$ and $E, 24$ h exposure to $A \beta$ was sufficient to induce a significant loss of spines in wild-type primary neurons $(0.28 \pm 0.075$ spines $/ \mu \mathrm{m})$ compared with WtCM treatment $(0.48 \pm 0.12$ spines $/ \mu \mathrm{m})$. However, no change of the dendritic complexity was observed, suggesting that such a short exposure with oligomeric $A \beta$ could not affect the whole dendritic arbor of the cells. A $\beta$ immunodepletion (using the antiamyloid- $\beta$ monoclonal antibody $6 \mathrm{E} 10)$ significantly inhibited the effect of TgCM on spines $(0.39 \pm 0.09$ spines $/ \mu \mathrm{m})$, demonstrating that the spine loss was directly related to the presence of amyloid $\beta$ species (immunodepletion led to a $70 \%$ decrease in TgCM A $\beta$ content). Remarkably, when cortical primary neurons were transfected with VIVIT-GFP, before exposure to TgCM, the effect on spine density was greatly ameliorated $(0.41 \pm 0.108$ spines $(\mu \mathrm{m})$, despite the persistence of $\mathrm{A} \beta$ species in the medium. This suggests that blocking CaN-mediated activation of NFAT protects against neurotoxic effects of soluble exogenous $A \beta$ oligomers.
A nuclear-targeted form of VIVIT, but not a membrane bound VIVIT, is a potent inhibitor of $\mathrm{CaN}$-induced morphological changes in primary neurons

VIVIT was initially identified as a selective blocker of the CaN/ NFAT interaction, mimicking the PxIxIT sequence of NFAT that is required to bind calcineurin. Although Aramburu et al. (1999) showed that calcineurin activity on critical substrates (such as CREB) was not affected by VIVIT, several recent studies suggested that it can also disrupt the interaction between $\mathrm{CaN}$ and other important cytosolic targets implicated in the regulation of synaptic activity such as AKAP or cabin (Dell'Acqua et al., 2002; Liu, 2003). In an attempt to precisely decipher the role of the transcription factor NFAT in A $\beta$-related neurotoxic events, we used a nuclear-targeted version of VIVIT that was shown to have a restricted localization within the nucleus and that was previously used to specifically counteract NFATc4-related transcriptional activity (Schwartz et al., 2009). As a negative control, we fused a myristoylation (Myr) tag to the VIVIT-GFP plasmid to direct the localization of this peptide toward the cell membrane. Thus, these constructs would disambiguate the transcriptional effects of VIVIT from other non-transcriptional functions at the spines.

We first tested whether these two constructs, NLS-VIVITGFP and Myr-VIVIT-GFP, showed the expected subcellular localization. At $5 \mathrm{DIV}$, primary cortical neurons were transfected with GFP, NLS-VIVIT-GFP, or Myr-VIVIT-GFP. As observed in Figure $4 A$, NLS-VIVIT-GFP was strictly restricted to the nuclear compartment and limited by the nuclear membrane marker laminin B1. By contrast, the fluorescent signal of Myr-VIVITGFP was completely excluded from the nucleus. When each plasmid was transfected altogether with DsRed (as a common denominator to obtain the full morphology of each cell), both GFP and Myr-VIVIT-GFP could be detected in the processes of neurons and in the dendritic spines, which was not the case for NLS-VIVIT-GFP (Fig. 4B).

To determine the effect of NLS-VIVIT and Myr-VIVIT on NFAT transcriptional activity, primary neurons were transduced with an AAV-pNFAT-Luc reporter vector, altogether with AAV$\mathrm{WtCaN}+\mathrm{GFP}, \mathrm{AAV}-\mathrm{CACaN}+\mathrm{AAV}-\mathrm{GFP}, \mathrm{AAV}-\mathrm{CACaN}+\mathrm{AAV}-$ NLS-VIVIT-GFP, or AAV-CACaN+AAV-Myr-VIVIT-GFP. After $3 \mathrm{~d}$, the luciferase activity was quantified. As previously observed, overexpression of a constitutively active form of calcineurin led to an increase of NFAT transcriptional activity compared with WtCaN. This increase was significantly diminished when NLSVIVIT-GFP was overexpressed, but no change could be detected with Myr-VIVIT-GFP. This suggested that only a nucleartargeted inhibitor was able to inhibit NFAT transcriptional activity in vitro (Fig. $5 A$ ). We next determined whether $\mathrm{CaN}$-induced morphological changes could be rescued by the different VIVITGFP constructs by co-transfecting neuronal cultures with either WtCaN or CACaN, altogether with GFP, NLS-VIVIT-GFP, or Myr-VIVIT-GFP. Because dendrites and spines were not detectable from the GFP signal when NLS-VIVIT-GFP was overexpressed, we used DsRed as a common denominator to evaluate spine density and dendritic complexity. At 18 DIV, both spine loss and dendritic simplification associated with CACaN overexpression were significantly improved when NLS-VIVIT-GFP was transfected (Fig. 5B-D). By contrast, no effect could be detected when Myr-VIVIT-GFP was used, consistent with the hypothesis that the neuroprotection of VIVIT against CACaN-mediated neurodegeneration is related to transcriptional effects in the nucleus rather than non-transcriptional effects in the cytoplasm or dendrites. 
A
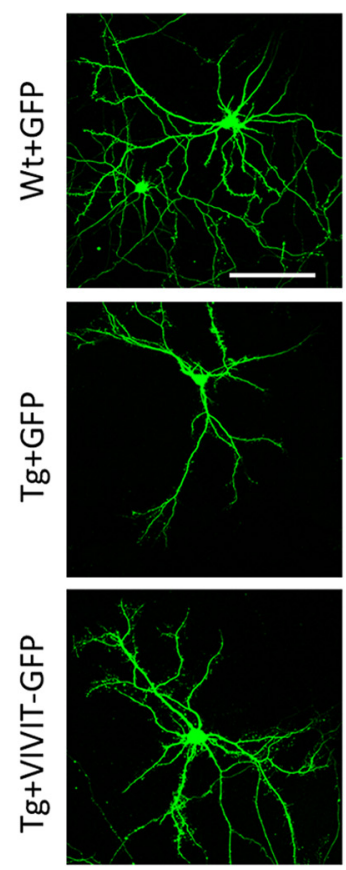

B

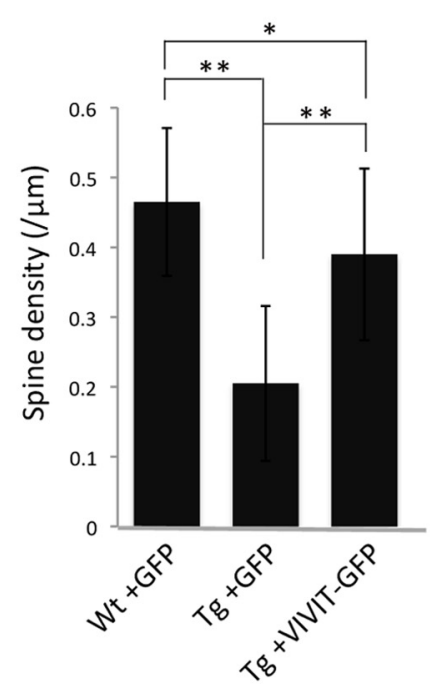

C
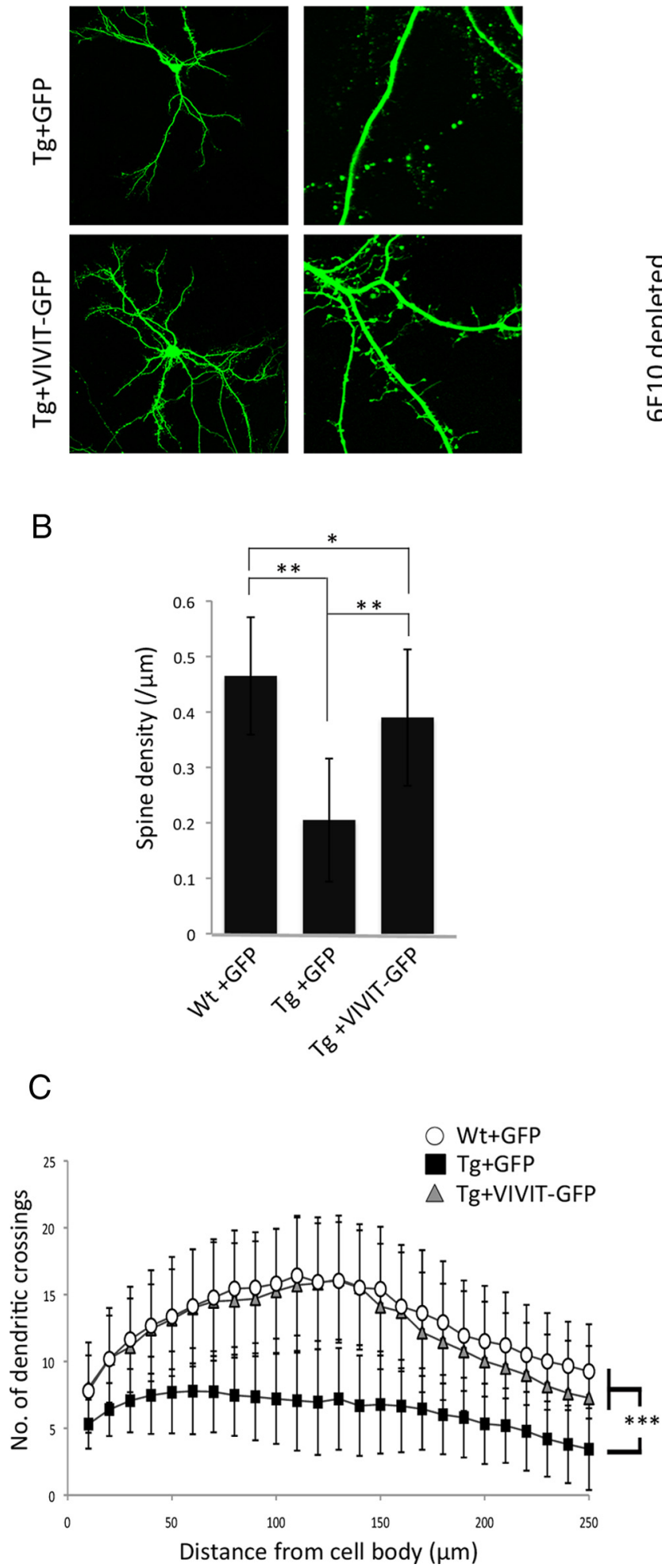

D
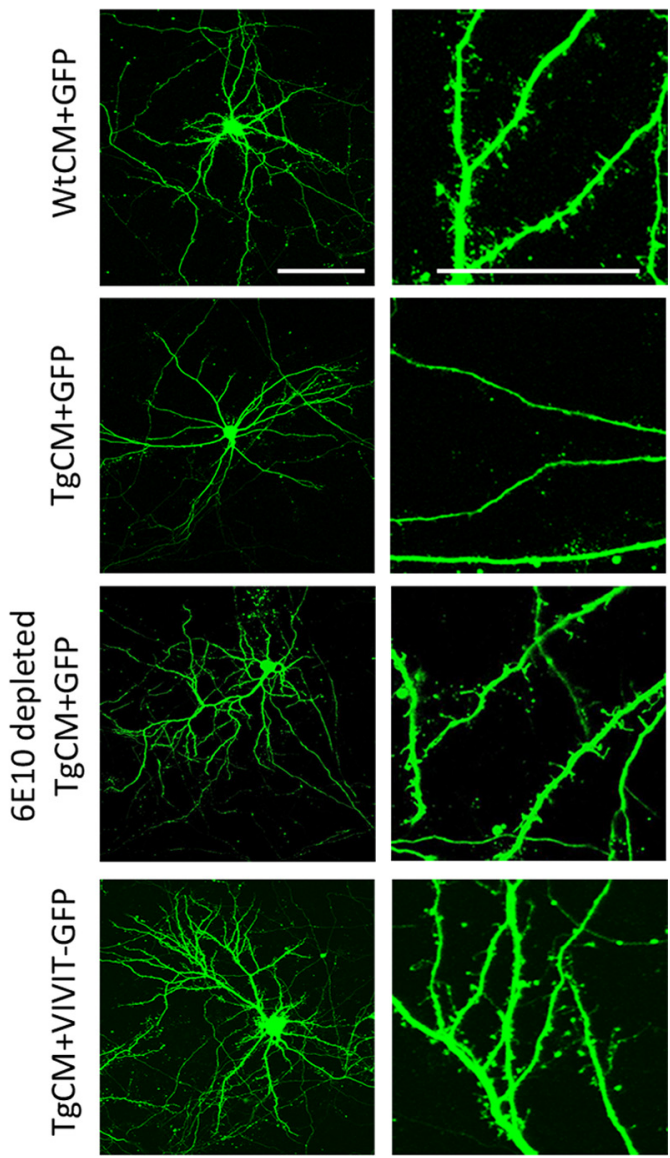

E

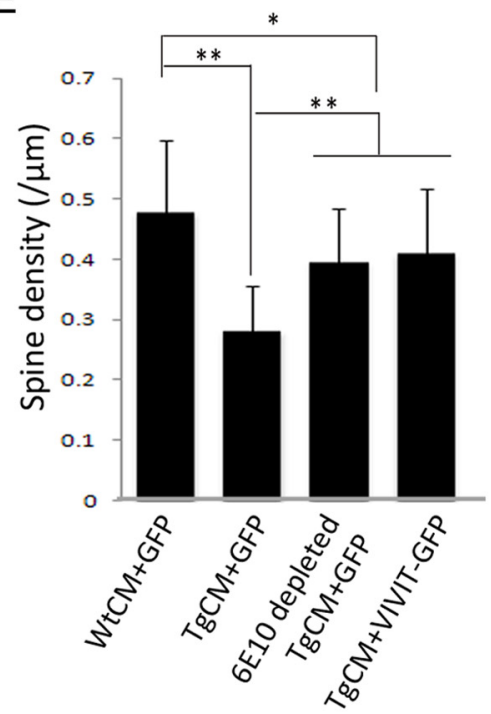

Figure 3. The morphological abnormalities observed in Tg neurons and in TgCM-treated neurons are improved by VIVIT-GFP. A, Representative images of Wt and Tg GFP-labeled neurons (left) and dendrites (right) at 18 DIV. Scale bar, $100 \mu \mathrm{m}$. B, GFP-transfected Tg neurons present a lower spine density compared with Wt littermate cells, but the number of spines is (Figure legend continues.) 
A

MERGE
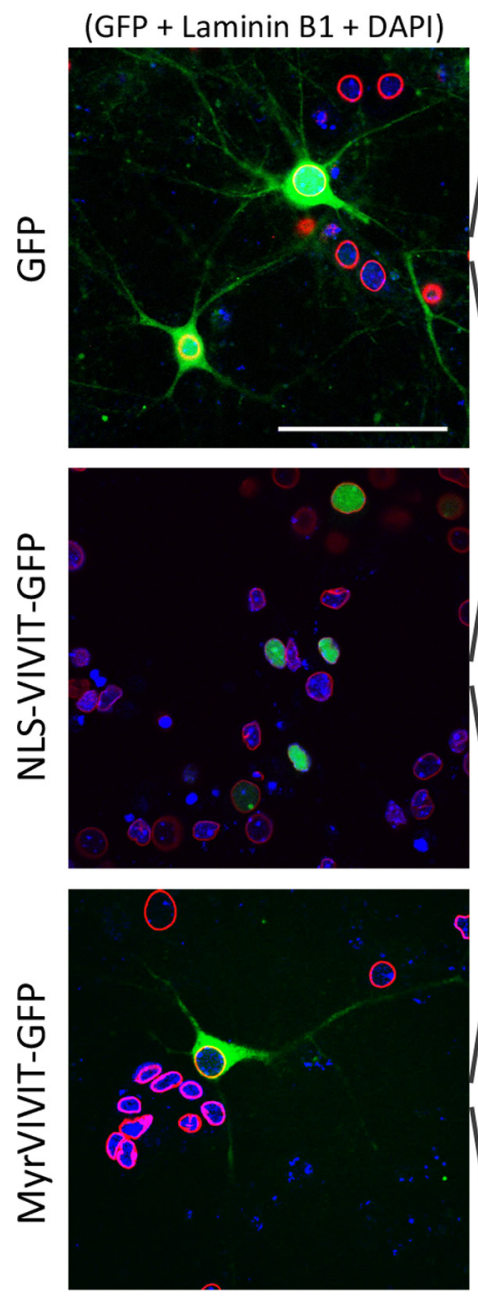
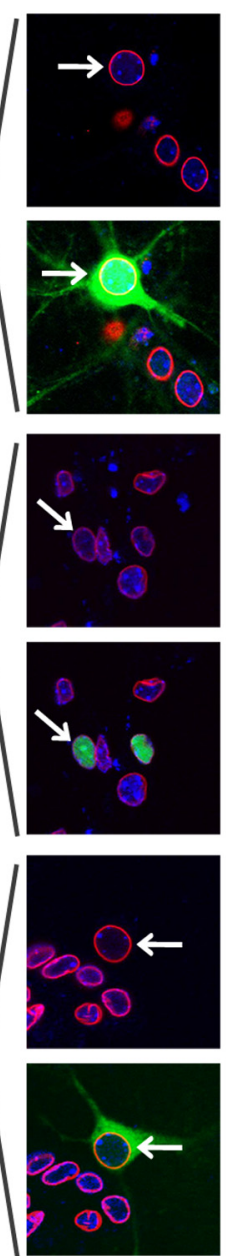

B

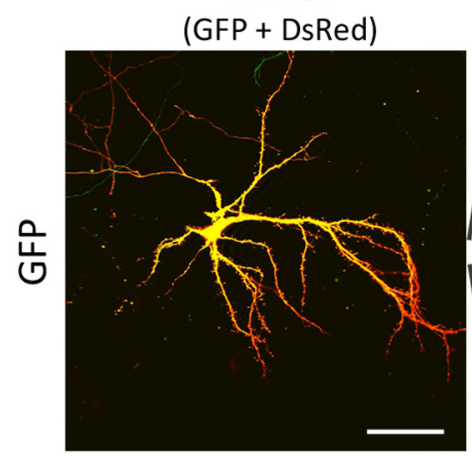

MERGE
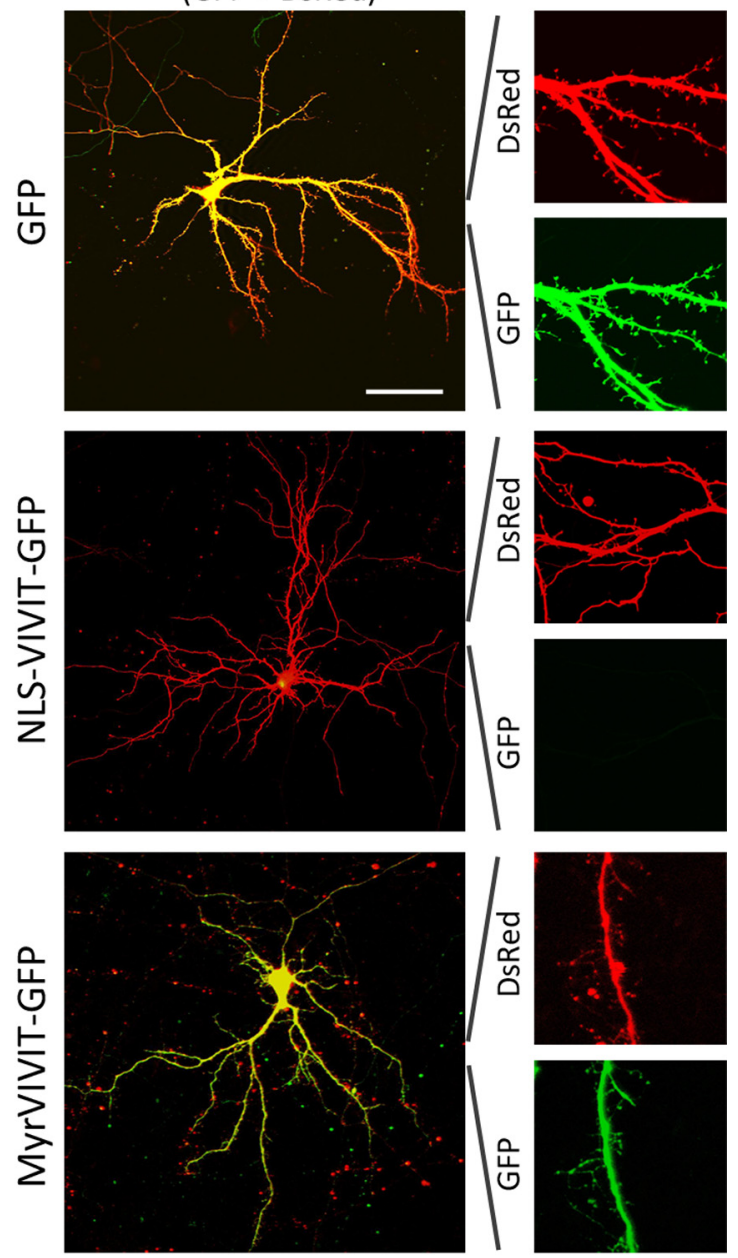

Figure 4. NLS-VIVIT-GFP localization is restricted to the nucleus, whereas Myr-VIVIT-GFP is excluded from this compartment. $A$, Representative images of primary neurons that were transfected with GFP, NLS-VIVIT-GFP, or Myr-VIVIT-GFP and then stained for laminin B1 and DAPI (left). On the right, higher magnification images show that GFP alone can be detected in the nuclear and cytoplasmic compartments, whereas NLS-VIVIT-GFP is surrounded by laminin B1 and colocalizes with DAPI, suggesting that it is restricted to the nucleus. Inversely, Myr-VIVIT-GFP is excluded from the nuclear compartment. Scale bar, 50 $\mu \mathrm{m} . \boldsymbol{B}$, Both GFP and Myr-VIVIT-GFP can be detected in the dendritic shaft and in spines, as suggesting by the colocalization with DsRed. NLS-VIVIT-GFP is, however, absent in the neuronal processes. Scale bar, $100 \mu \mathrm{m}$.

Nuclear-targeted VIVIT, but not membrane-targeted VIVIT, protects against $\mathrm{A} \boldsymbol{\beta}$ neurotoxicity in primary neurons

In the previous experiment, we determined that a nuclear version of VIVIT was almost as potent as a nontargeted VIVIT at blocking the pathological changes induced by CACaN. Considering the fact that calcium dyshomeostasis and $\mathrm{CaN}$ activation have been shown to be downstream events to $\mathrm{A} \beta$ neurotoxicity (Busche et al., 2008;

\section{$\leftarrow$}

(Figure legend continued.) significantly improved when Tg neurons were previously transfected with VIVIT-GFP. C, In addition, Sholl's plot analyses of Wt and Tg cultures show an overall decreased complexity of the dendritic arborization in Tg neurons overexpressing GFP compared with Wt neurons. The simplification of the dendritic arbor is fully corrected when Tg neurons were transfected by VIVIT-GFP and it reaches the level of complexity observed in Wt neurons ( $p=$ NS compared with Wt neurons transfected with GFP). D, Representative images of wild-type cortical neurons (left) and dendrites (right) expressing GFP or VIVIT-GFP after treatment for $24 \mathrm{~h}$ with WtCM, TgCM, or depleted $\mathrm{TgCM}$. Scale bar, $100 \mu \mathrm{m}$. E, Dendritic spine quantification demonstrates that a short exposure to oligomeric $A \beta$ leads to a significant decrease of their density, whereas spines are unaffected when WtCM was applied. However, the spine density is maintained when $\mathrm{TgCM}$-treated neurons overexpress VIVIT-GFP or when $A \beta$ peptides were previously immunodepleted from the medium using the $6 \mathrm{E} 10$ antibody. ${ }^{*} p<0.01,{ }^{* *} p<0.0001$ and ${ }^{* * *} p<10^{-10}$. (n>40 cells per condition).
Kuchibhotla et al., 2008), we next asked whether a nuclear-targeted version of this peptide may be protective against $\mathrm{A} \beta$ toxicity as well.

Following the same procedures as described above, we tested the NLS-VIVIT-GFP or Myr-VIVIT-GFP constructs in Wt and Tg primary neurons that were co-transfected with DsRed (Fig. 6A). Both spine density and dendritic complexity were significantly improved when NLS-VIVIT-GFP was overexpressed in Tg neurons, but no improvement was detected with Myr-VIVIT-GFP (Fig. 6B,C).

Similarly, when Wt primary neurons were transfected with GFP+DsRed，NLS-VIVIT-GFP+DsRed，or Myr-VIVIT-GFP+ DsRed and then treated for $24 \mathrm{~h}$ with $\mathrm{A} \beta$-containing TgCM (Fig. $6 D)$, the TgCM-induced spine loss was attenuated by NLS-VIVITGFP (Fig. 6E), but no change could be observed in TgCM-treated neurons transfected with GFP or Myr-VIVIT-GFP. Again, this short-term exposure to $A \beta$ oligomers did not affect the dendritic branching of the cortical neurons (data not shown).

Together, these observations strongly suggest that NFAT-related transcriptional events play a role in $\mathrm{A} \beta$-related neurotoxicity, thus predicting that inhibition of the CaN/NFAT pathway may be a protective strategy against $\mathrm{A} \beta$-induced neuronal injuries in vivo. 
A

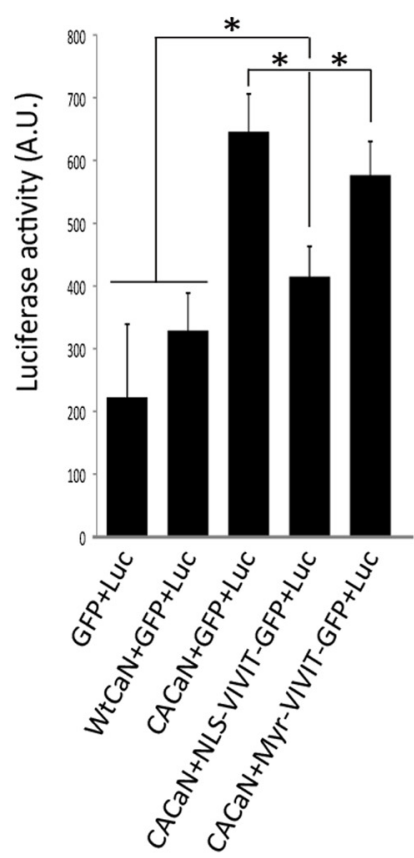

C

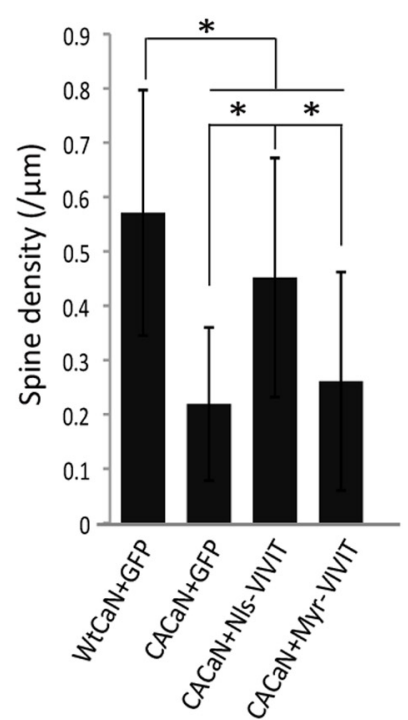

D
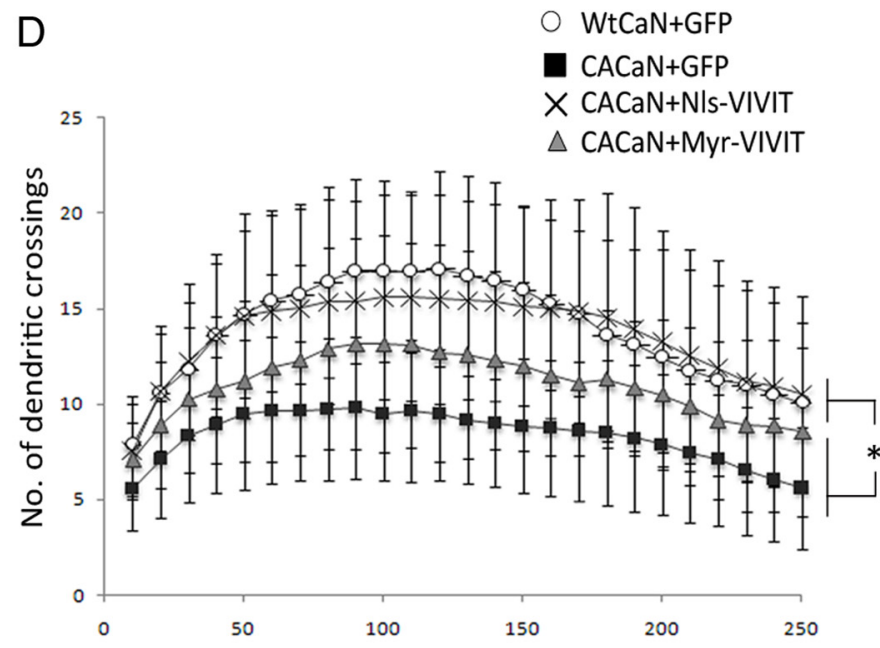

Distance from cell body $(\mu \mathrm{m})$
B
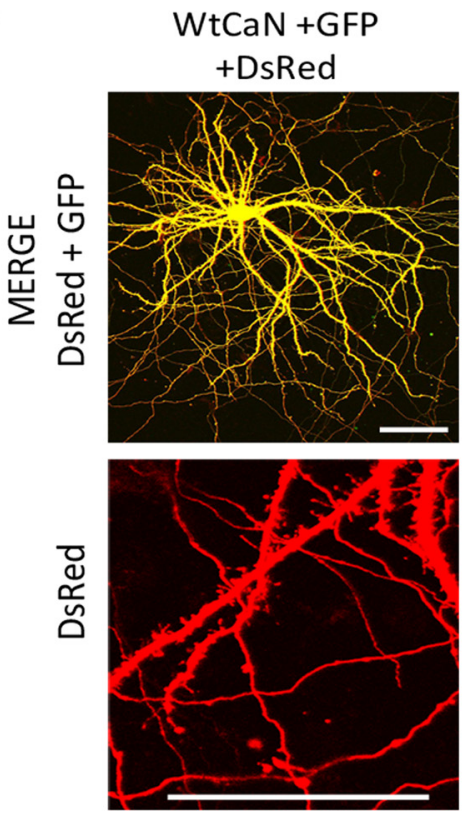

CACaN+GFP +DsRed
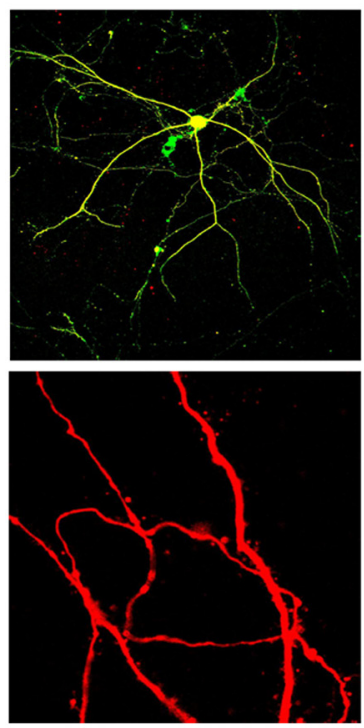

CACaN+NIs-VIVIT + DsRed
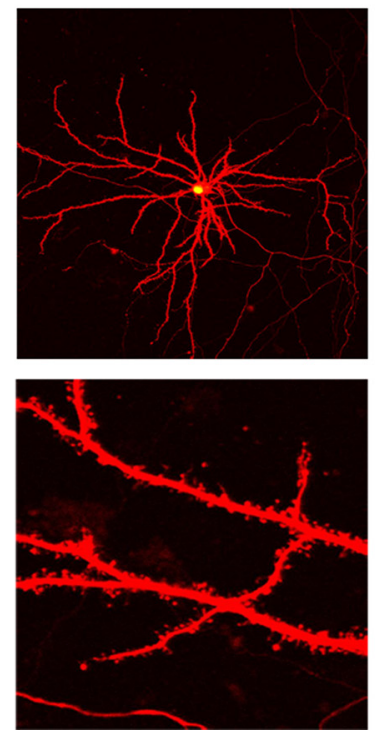

CACaN+Myr-VIVIT + DsRed
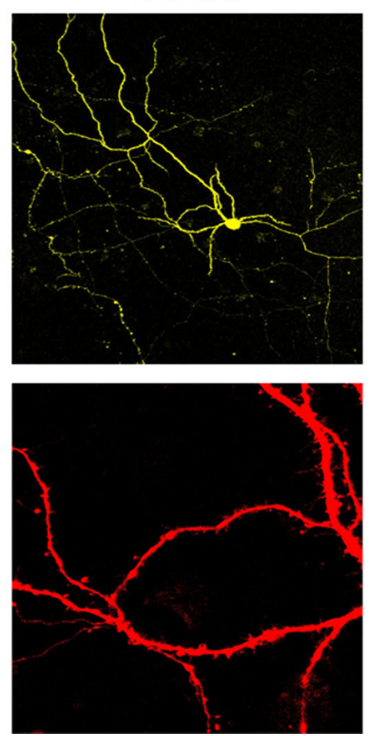

Figure 5. The restricted localization of VIVIT into the nucleus potently inhibits the morphological abnormalities induced by an overactivated form of CaN. A, NFAT transcriptional activity assessed using the pNFAT-luciferase reporter system shows that overexpression of NLS-VIVIT-GFP, altogether with CACaN, is associated with a decreased luciferase activity. No effect is observed when Myr-VIVIT-GFP is overexpressed, suggesting that this inhibitor does not affect NFAT transcriptional activity. $\boldsymbol{B}$, Representative images of cortical primary neurons (top) and dendrites (bottom) that were co-transfected with GFP, NLS-VIVIT-GFP, and Myr-VIVIT-GFP altogether with CACaN and WtCaN. To be able to observe the neurites and quantify the dendritic spines in all the conditions, cells were co-transfected with a DsRed fluorescent reporter. Scale bar, $100 \mu \mathrm{m}$. C, The decreased spine density associated with the overexpression of a constitutively activated CaN (CACaN) can be improved by co-transfection with a nuclear-targeted VIVIT (NLS-VIVIT-GFP), whereas no beneficial effects is observed with a membrane-bound VIVIT. D, Overexpression of NLS-VIVIT also leads to a more complex dendritic arborization of CACaN transfected neurons, whereas overexpression of Myr-VIVIT cannot rescue the effects of CACaN. ${ }^{*} p<0.05$. ( $n>30$ cells per condition).

\section{Disruption of NFAT pathway with VIVIT and NLS-VIVIT} increases spine density in the vicinity of amyloid plaques in vivo

In vitro, we observed that a selective disruption of the interaction between $\mathrm{CaN}$ and NFAT may alleviate $\mathrm{A} \beta$-related neurotoxicity. We asked whether these neuroprotective effects could be reproduced in vivo. Neurites surrounding amyloid deposits have an increased calcium overload, decreased spine density, and an increased probability to develop neuritic dystrophies (Spires et al., 2005; Kuchibhotla et al., 2008). Interestingly, overexpression of a constitutively activated $\mathrm{CaN}$ in vivo leads to similar abnormal neuronal changes (Wu et al., 2010). We therefore hypothesized that amyloid aggregates may be responsible for an abnormal increased activation of CaN/NFAT pathway in vivo, which would compromise neuronal integrity. We revisited this hypothesis by delivering VIVIT in the brains 
A MERGE GFP +DsRed
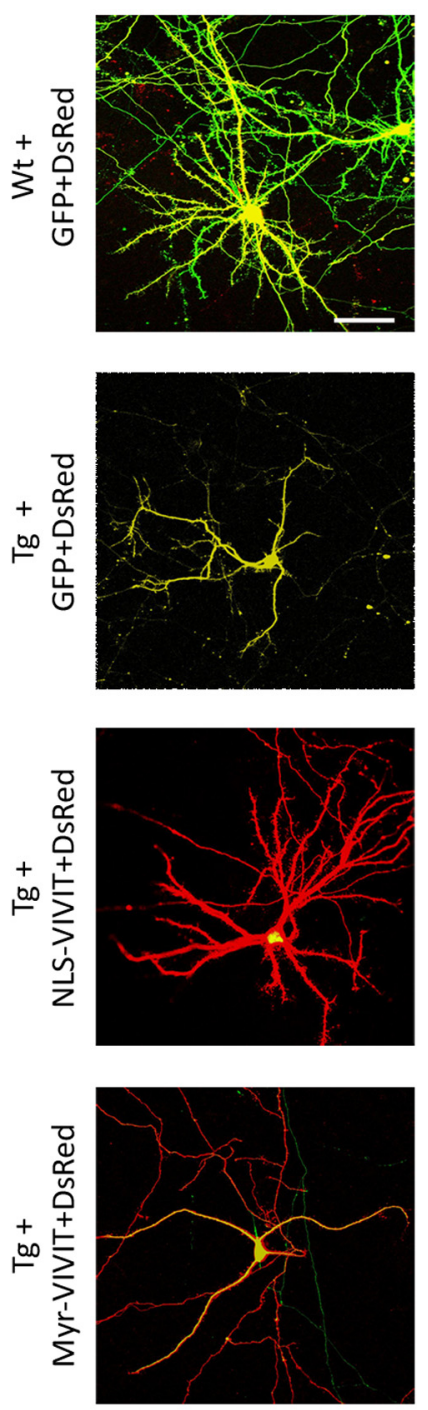

B

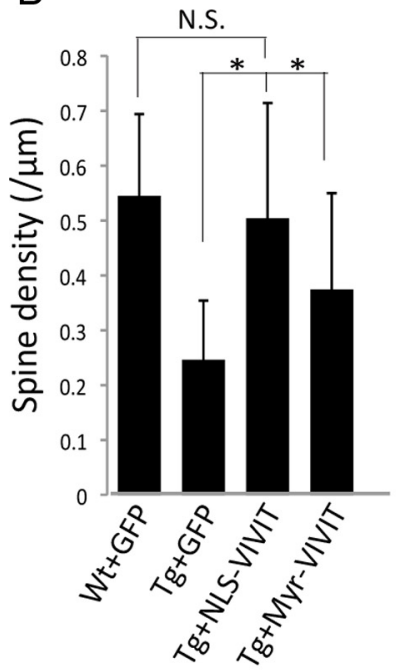

DsRed
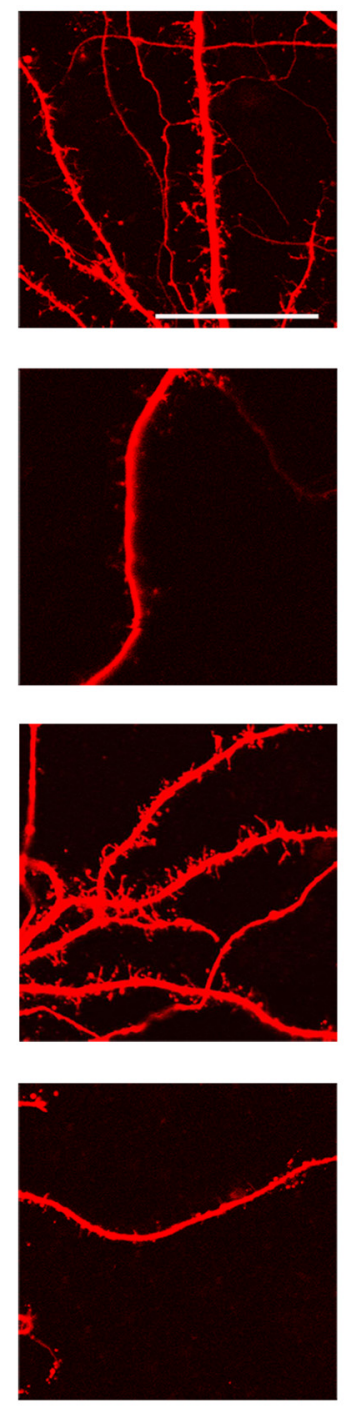

C

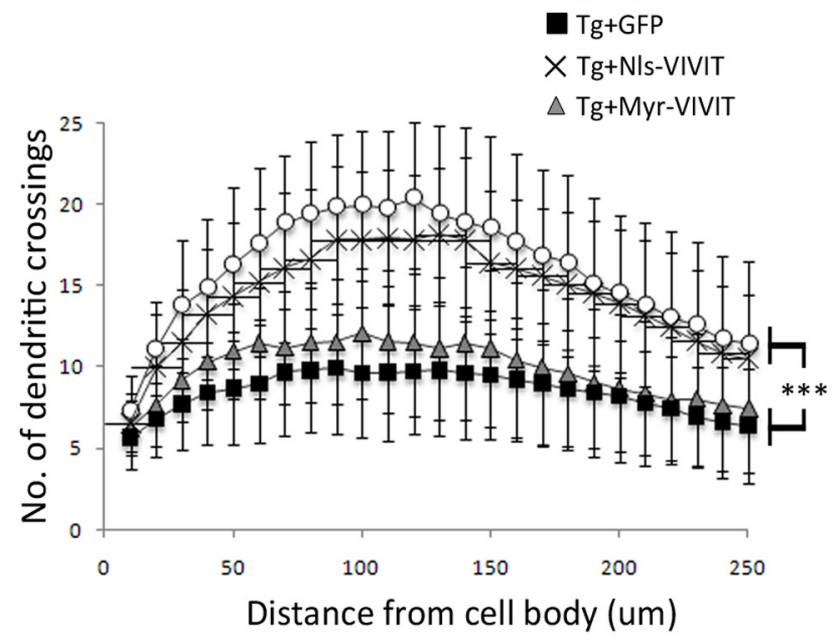

MERGE

GFP +DsRed
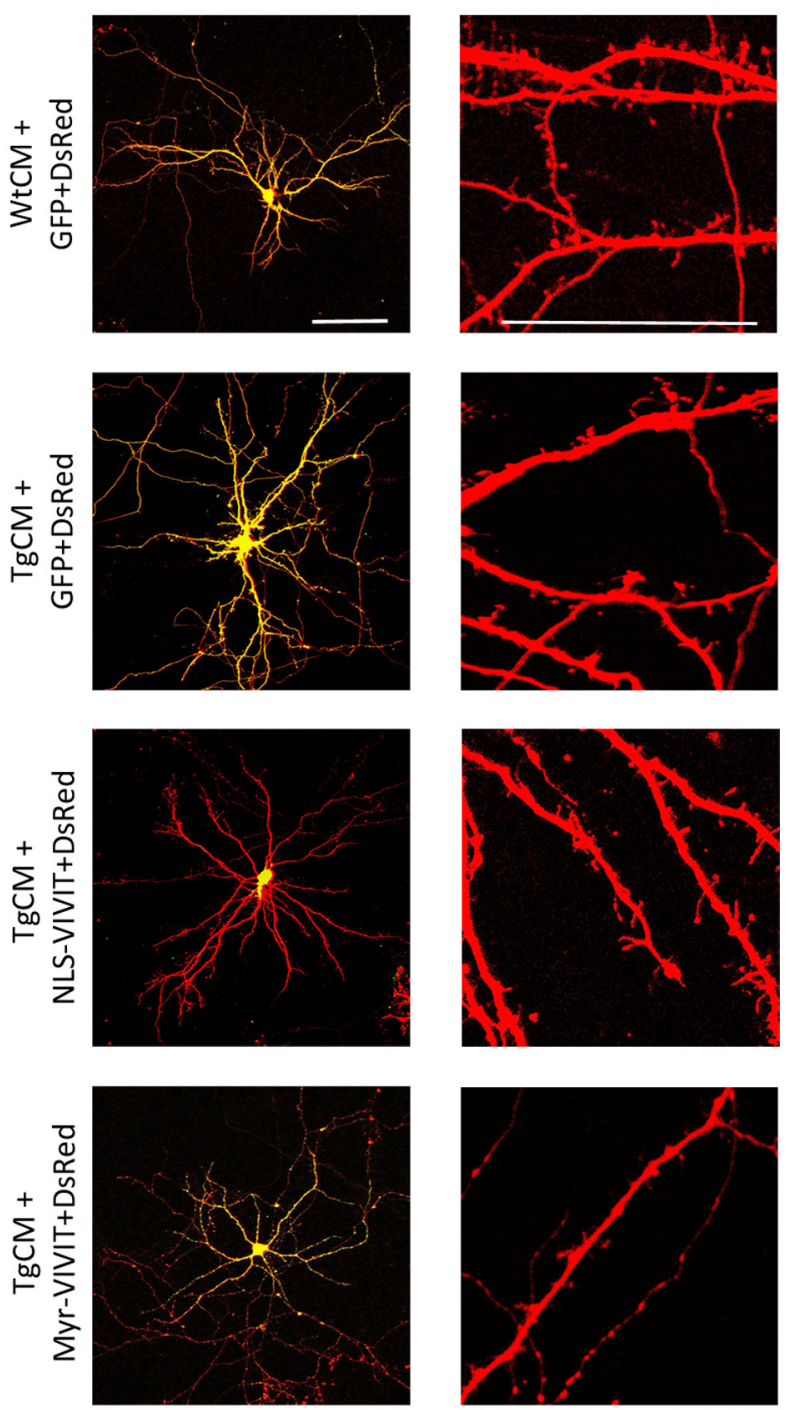

E

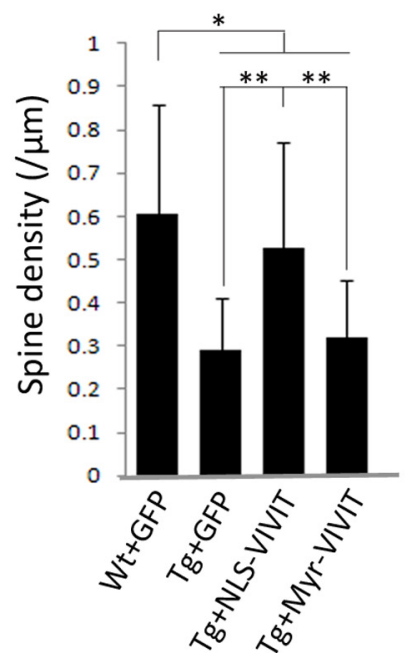

Figure 6. Aberrant neuronal morphology observed in Tg neurons and in TgCM-treated cells are prevented by NLS-VIVIT-GFP but not by Myr-VIVIT-GFP. A, Representative images of DsRed-filled Wt or Tg neurons (left) and dendrites (right) overexpressing GFP or the different VIVIT constructs show that Tg cells transfected by NLS-VIVIT-GFP present an increased spine density compared with GFP transfected Tg cells. Scale bar, $100 \mu \mathrm{m}$. B, Bar graph summarizing the averaged spine density of each group confirms that only the nuclear targeted peptide is able to improve the morphological abnormalities observed in Tgneurons, but this is not the case when Myr-VIVIT-GFP is overexpressed. C, Sholl's plot analysis demonstrates that NLS-VIVIT-GFP also increases the dendritic (Figurelegend continues.) 
of APP/PS1 transgenic mice and examining its effects on neuritic degeneration.

To investigate the effect of VIVIT in vivo, AAV vectors encoding for VIVITGFP, NLS-VIVIT-GFP (both inhibitors having proven beneficial effects in vitro), and GFP (as a control) were stereotactically injected in the cortex of 7-month-old littermates or APP/PS1 mice, when amyloid deposits are already present. In the case of AAV-NLS-VIVIT-GFP injected mice, we first verified that the recombinant protein was properly addressed to the nuclear compartment and colocalized with Hoechst-stained nuclei (Hoechst solution was applied topically; Fig. 7A). AAV-NLS-VIVIT-GFP and AAV-GFP were then coinjected with a ratio of $3: 1$, so that a high proportion of GFP filled neurons were also transduced by the AAVNLS-VIVIT-GFP vector. One month later, a cranial window was implanted. Neurites and spines were detected and quantified by multiphoton imaging in the living animal (Fig. 8A). Highmagnification images were taken to visualize dendritic spines. Spine density was quantified for each neuritic segment using the NeuronStudio software (see Materials and Methods). Importantly, no significant difference was observed when comparing the spine density in wild-type littermate mice injected with either AAV-GFP $(0.47 \pm 0.11$ spines $/ \mu \mathrm{m})$, AAV-VIVIT-GFP (0.49 \pm 0.107 spines/ $\mu \mathrm{m})$, or AAV-NLS-VIVIT-GFP (0.53 \pm 0.1 spines $/ \mu \mathrm{m}$ ) (data not shown), indicating that VIVIT-GFP does not have an effect on spine density by itself, i.e., independently of $A \beta$.

Compared with dendrites from GFP-injected wild-type littermates $(0.47 \pm 0.11$ spines $/ \mu \mathrm{m})$, dendrites in the vicinity of amyloid deposits ( $<100 \mu \mathrm{m}$ away from plaque) in APP/PS1 mice exhibited a decreased spine density $(0.31 \pm 0.12$ spines $/ \mu \mathrm{m})$. This amyloid-associated spine loss was restored to essentially normal levels when either AAV-VIVIT-GFP $(0.49 \pm 0.13$ spines/ $\mu \mathrm{m})$ or AAV-NLS-VIVIT-GFP+AAV-GFP $(0.44 \pm 0.1$ spines/ $\mu \mathrm{m}$ ) were injected in APP/PS1 mice (Fig. $8 B$ ). In both APP/PS1 animals and human $\mathrm{AD}$ brains the spine densities are correlated with the distance from the edge of the amyloid deposits (correlation coefficient: 0.41) (Koffie et al., 2009) (Spires et al., 2005). However, this local effect of amyloid plaques was nearly abolished when AAV-NLS-VIVIT-GFP (correlation coefficient: 0.22 ) or AAV-VIVIT-GFP was expressed (correlation coefficient: 0.09; Fig. 8C).

$\leftarrow$

(Figure legend continued.) complexity compared with GFP-transfected Tg neurons and reaches the level of complexity observed in Wt neurons. $D$, Images representative of DsRed filled neurons (left) or dendritic segments (right) after co-transfection by GFP, NLS-VIVIT-GFP, or Myr-VIVIT-GFP and $24 \mathrm{~h}$ treatment with WtCM and TgCM. Scale bar, $100 \mu \mathrm{m}$. E, A decreased spine density was induced by $\mathrm{TgCM}$, which could be inhibited by NLS-VIVIT-GFP but not by Myr-VIVIT-GFP. ${ }^{*} p<0.05,{ }^{* *} p<0.001$ and ${ }^{* * *} p<10^{-5}$. ( $n>40$ cells per condition).
Overall, the injection of AAV-VIVIT-GFP was associated with a marked recovery of spine density around amyloid deposits in vivo. A significant beneficial effect was also observed in AAVNLS-VIVIT injected mice, even though this improvement did not reach the levels of VIVIT-GFP. This difference might be due to the fact that some of the GFP-filled neurites observed in animals coinjected with AAV-NLS-VIVIT-GFP and AAV-GFP had only been transduced by the later vector. To examine this possibility, we coinjected the AAV-NLS-VIVIT-GFP and an AAVTdTomato with the same 3:1 ratio as previously used, and we observed that $86 \%$ of the red fluorescent cells also contained a nuclear GFP signal (Fig. 7B), suggesting that the vast majority of transduced neurons expressed both recombinant proteins.

\section{Neuritic abnormalities observed around amyloid deposits in APP/PS1 mice are partially restored by VIVIT-GFP and NLS-VIVIT-GFP}

The presence of amyloid deposits not only affects the density of dendritic spines, but is also known to disturb the morphology of the neuritic shaft itself (Knowles et al., 1999). We therefore asked whether overexpression of VIVIT or NLS-VIVIT might have an additional rescue effect in regards to these morphological parameters.

As previously shown, amyloid deposits are associated with the development of neuritic dystrophies, which can be visualized by AAV-GFP injection in APP/PS1 mice. Even though the size of the 

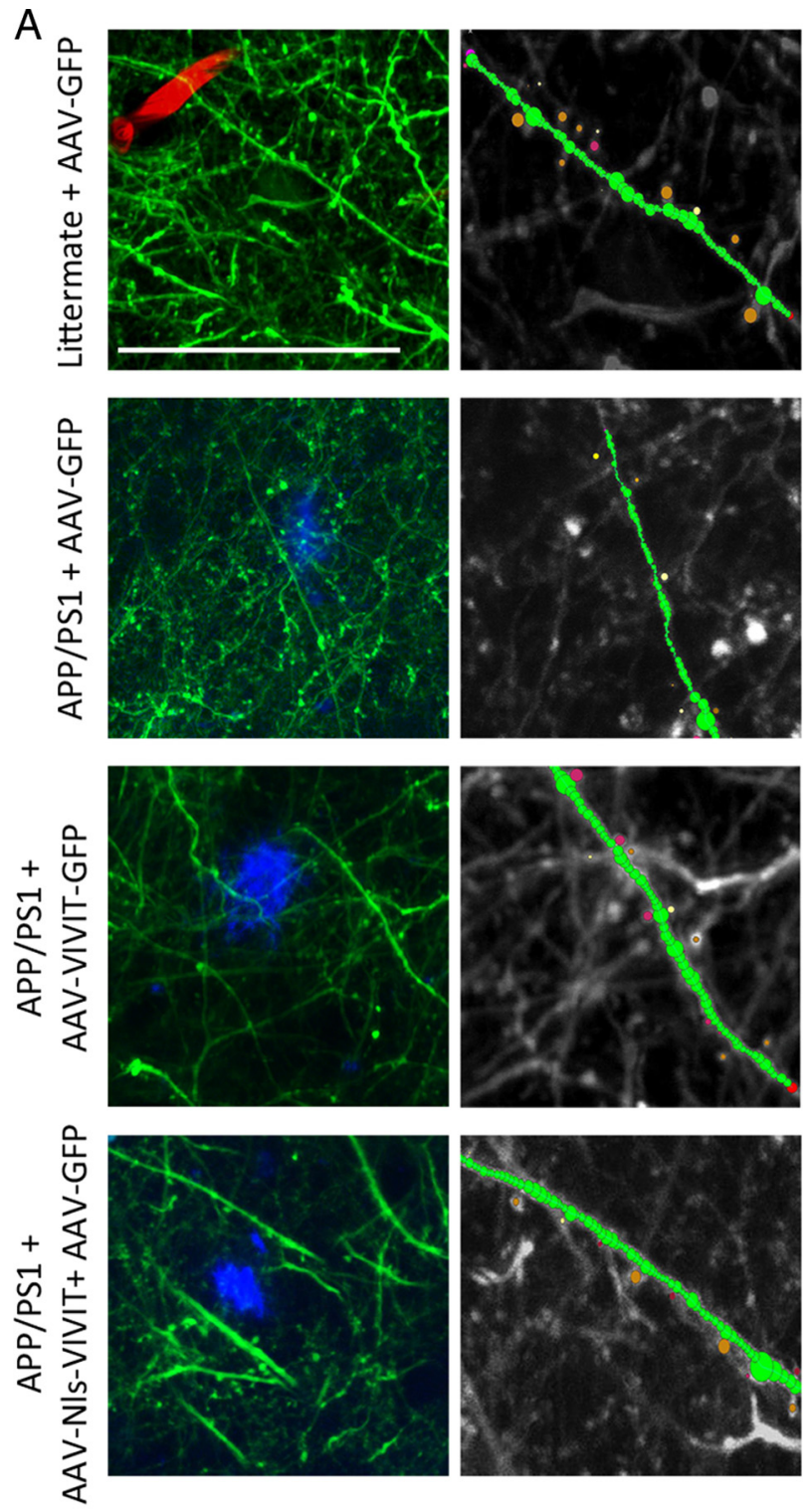

B
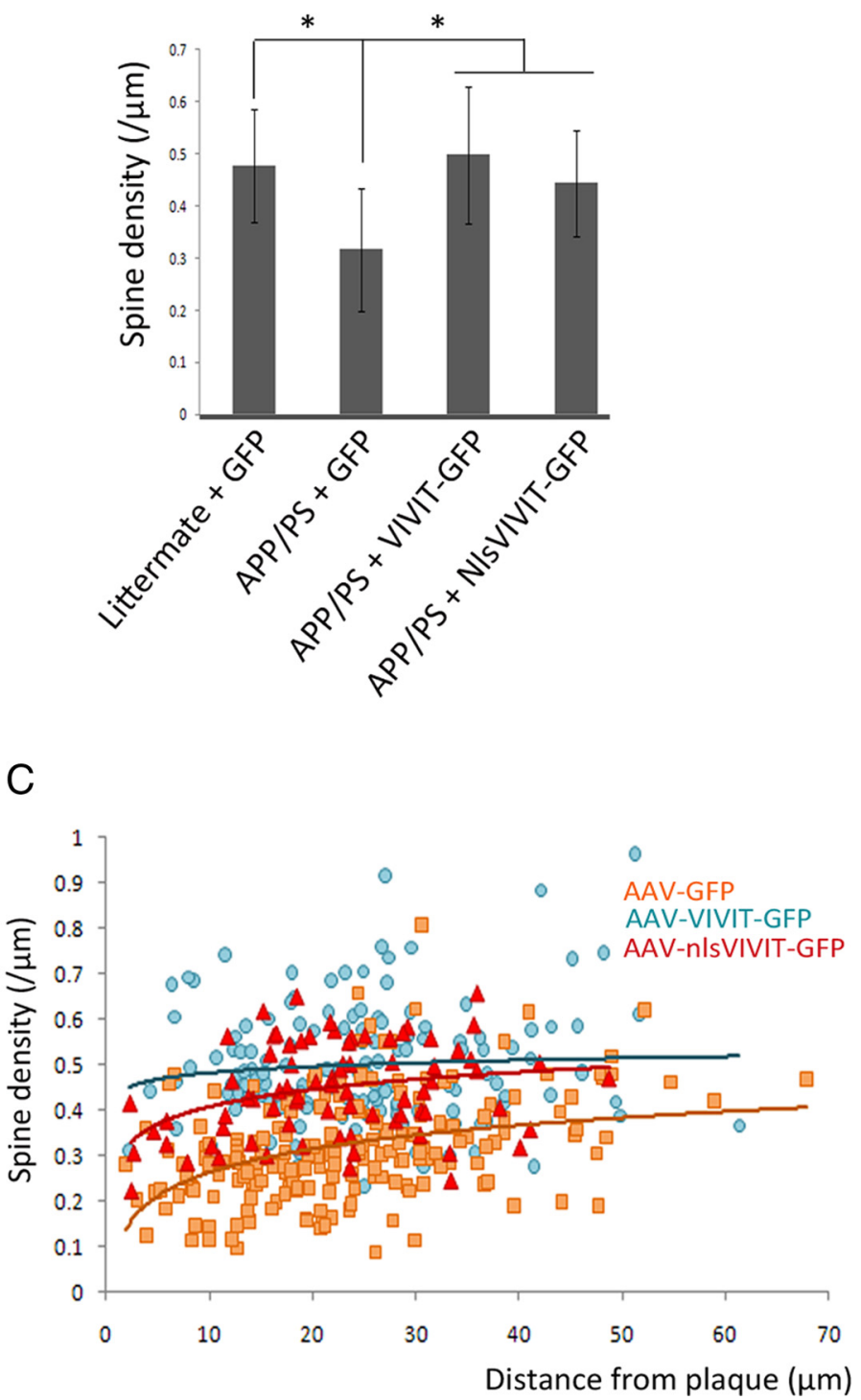

Figure 8. Spine loss detected in the vicinity of amyloid deposits is significantly improved by VIVIT-GFP and NLS-VIVIT-GFP. A, Left, z-projections of two-photon images representative of GFP-filled neurites in littermate mice injected with AAV-GFP or surrounding amyloid deposits in APP/PS1 mice injected with AAV-GFP, AAV-VIVIT-GFP, and AAV-NLS-VIVIT-GFP + AAV-GFP. Right, Pictures represent an example of dendritic spine detection using the Neuronstudio software. Brown, red, and yellow circles respectively indicate mushroom, stubby, and thin spines. Scale bar, $50 \mu \mathrm{m} . \boldsymbol{B}$, Bar graph summarizing the averaged spine density of each group confirms that APP/PS1 mice injected with AAV-VIVIT-GFP and AAV-NLS-VIVIT-GFP have a higher spine density than AAV-GFP injected Tg animals within $100 \mu \mathrm{m}$ surrounding amyloid deposits. Spine densities of either VIVIT-GFP or NLS-VIVIT-GFP treated groups are not significantly different from GFP injected littermates. C, Scatter plot of spine density data collected from GFP-injected APP/PS1 mice show a positive correlation between spine density and distance from amyloid deposit, but this local effect of amyloid plaque is partially and even completely abolished when animals were treated with NLS-VIVIT-GFP or VIVIT-GFP, respectively. ${ }^{*} p<0.05$ between AAV-GFP-treated APP/PS1 mice and either AAV-VIVIT-GFP or AAV-NLS-VIVIT-GFP injected animals. ( $n=6$ animals and $n<200$ dendrites analyzed per condition).

amyloid plaques that were analyzed was comparable between all the injected animals, plaques from AAV-VIVIT-treated mice were associated with fewer dystrophies (5 \pm 4 dystrophies/ plaque) compared with those from AAV-GFP (14 \pm 9 dystrophies/plaque) and AAV-NLS-VIVIT (18 \pm 10 dystrophies/ plaque; Fig. 9A,B) injected mice. Because the density of GFPfilled neurons around plaques was similar among all the groups (data not shown), we conclude that the difference in the number of dystrophies was not due to a decreased amount of GFP-filled neurites between AAV-GFP and AAV-VIVIT-GFP treated animals. This finding suggests that dystrophies, unlike dendritic spine loss, might not be recovered by a restricted inhibition of NFAT transcriptional activity in the nucleus. Interestingly, like
VIVIT, calcineurin inhibition with FK506 led to an improvement in neuritic dystrophies (Rozkalne et al., 2011). The remaining dystrophies in AAV-VIVIT-treated mice were similar in size to those from AAV-GFP and AAV-NLS-VIVIT-injected mice.

A third morphological characteristic of neurites around amyloid deposits is a subtle change in their trajectories (Knowles et al., 1999). This abnormal neuritic curvature was assessed on paraffin-embedded sections after immunostaining for GFP and amyloid plaques. The curvature ratio was calculated by reporting the length of a neurite divided by the end-to-end length of the same segment. We observed that the average neuritic curvature of both AAV-VIVIT-GFP $(1.054 \pm 0.038)$ and AAV-NLS-VIVITGFP (1.059 \pm 0.048$)$ injected mice were improved around amy- 
A

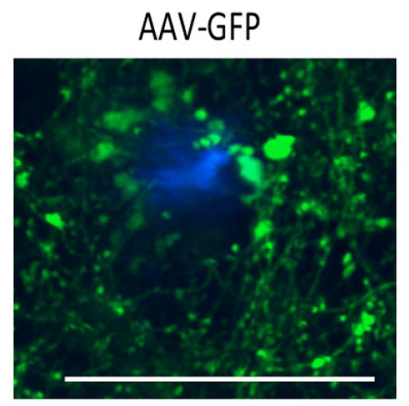

C

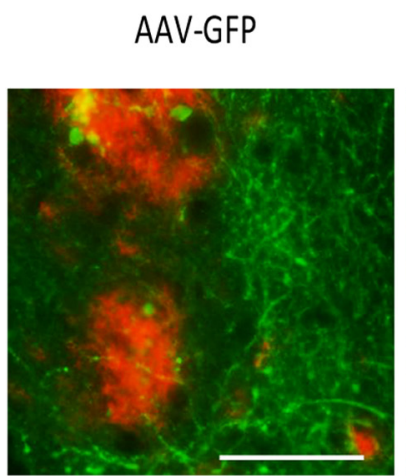

D

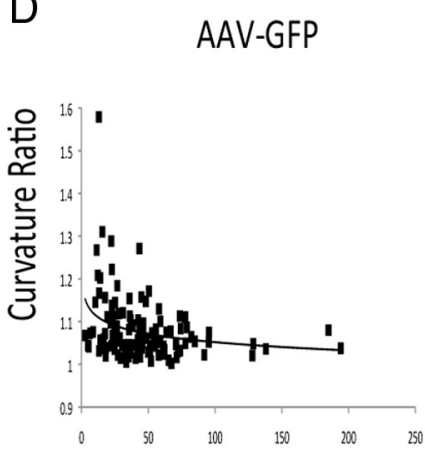

AAV-VIVIT-GFP

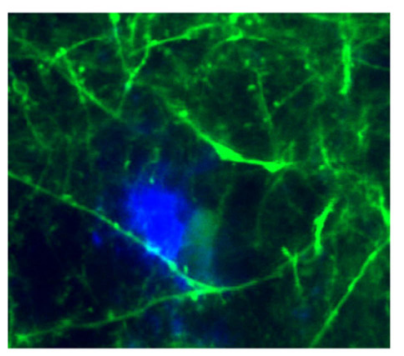

AAV-VIVIT-GFP

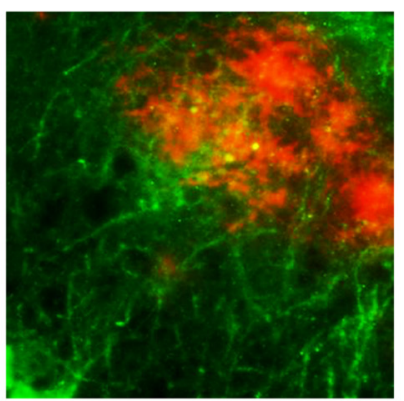

AAV-VIVIT-GFP
AAV-NLS-VIVIT-GFP +

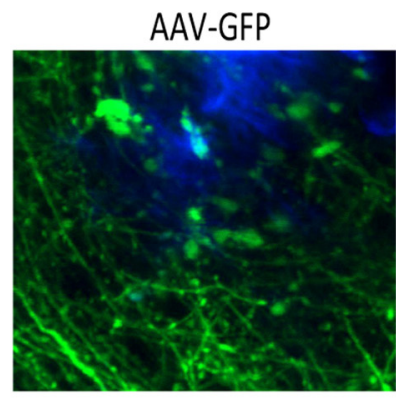

AAV-NLS-VIVIT-GFP + AAV-GFP

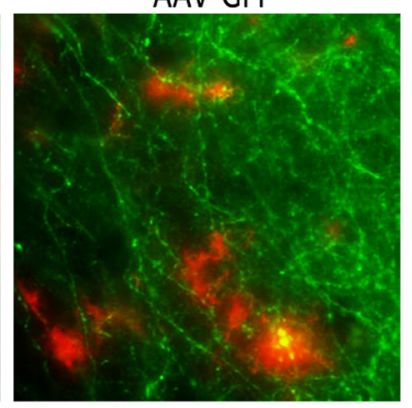

AAV-NLS-VIVIT-GFP + AAV-GFP

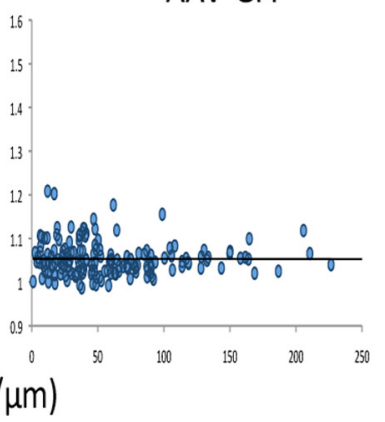

B

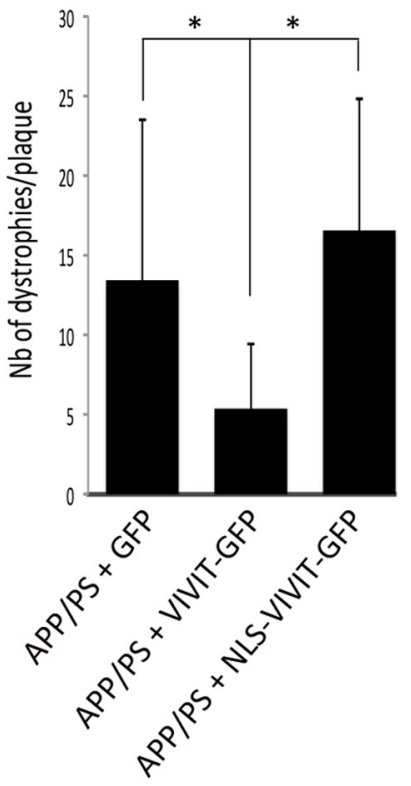

Figure 9. Overexpression ofVIVIT-GFP and NLS-VIVIT-GFP partially improve the neuriticabnormalities in vivo. Representative pictures $(\boldsymbol{A})$ and quantifications ( $\boldsymbol{B}$ ) of dystrophies surrounding amyloid deposits show that fewer neuritic dystrophies are present when AAV-VIVIT-GFP is injected, but no improvement can be observed when NLS-VIVIT-GFP is overexpressed. Scale bar, $50 \mu \mathrm{m}$. $(n<35$ amyloid plaques and $n<250$ dystrophies analyzed per group). C, Representative images of GFP and amyloid deposits immunostaining in APP/PS1-injected mice. Scale bar, $100 \mu \mathrm{m}$. D, In APP/PS1 mice injected with AAV-GFP, the increased curvature ratio is inversely correlated with the distance of each neurite to the closestamyloid deposit $(p<0.0001$, slope $=-0.0006)$. There is, however, no such correlation in APP/PS1 mice treated with either VIVIT-GFP $\left(p=0.953\right.$, slope $\left.=-5.12 \times 10^{-6}\right)$ or NLS-VIVIT-GFP $(p=0.812$, slope $=0.00003) .{ }^{*} p<0.05$. $(n=6$ animals per group $)$.

loid deposits compared with AAV-GFP-treated animals (1.081 \pm 0.07) (Fig. 9C,D).

\section{The beneficial effects associated with VIVIT-GFP and} NLS-VIVIT-GFP correlate with a decreased recruitment of NFATc4 into the nucleus

Using an in vivo imaging approach, we observed that several neuronal morphological parameters (i.e., spine density, neuritic dystrophies and curvature) that are abnormal in the vicinity of amyloid deposits were significantly improved when either AAVVIVIT-GFP or AAV-NLS-VIVIT-GFP was injected in the cortex of APP/PS1 mice. To verify that these beneficial effects were related to the efficient inhibition of NFATc4 recruitment into the nucleus, we performed a postmortem immunohistological analysis.

Brain sections of injected mice were stained to detect both GFP and endogenous NFATc4 to determine the ratio of NFATc4 in the nucleus versus cytoplasm in transduced neurons. We found that the distribution of NFATc4 nuclear/cytoplasmic ratios was shifted toward lower values in APP/PS1 mice injected with AAV-VIVIT-GFP treated animals $(0.8 \pm 0.08)$ compared with AAV-GFP $(0.89 \pm 0.06)$. AAV-NLS-VIVIT-GFP-injected mice exhibited an intermediate NFATc4 nuclear/cytoplasmic ratio between AAV-GFP and AAV-VIVIT-GFP-injected animals $(0.83 \pm 0.09)$ (Fig. $10 A, B)$.

\section{Discussion}

The biological mechanisms that sculpt the fine structure of the adult brain and their alterations in neurodegenerative diseases remain largely unknown. In this report we demonstrated that $\mathrm{A} \beta$ neurotoxic damage can be prevented or even reversed by inhibiting calcineurin-mediated activation of NFAT. Importantly, the same profound morphological changes occur when a constitu- 
A

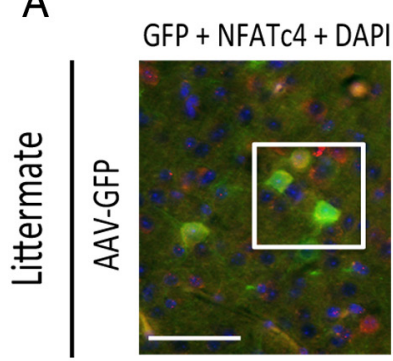

NFATC4 + DAPI
(higher magnification)
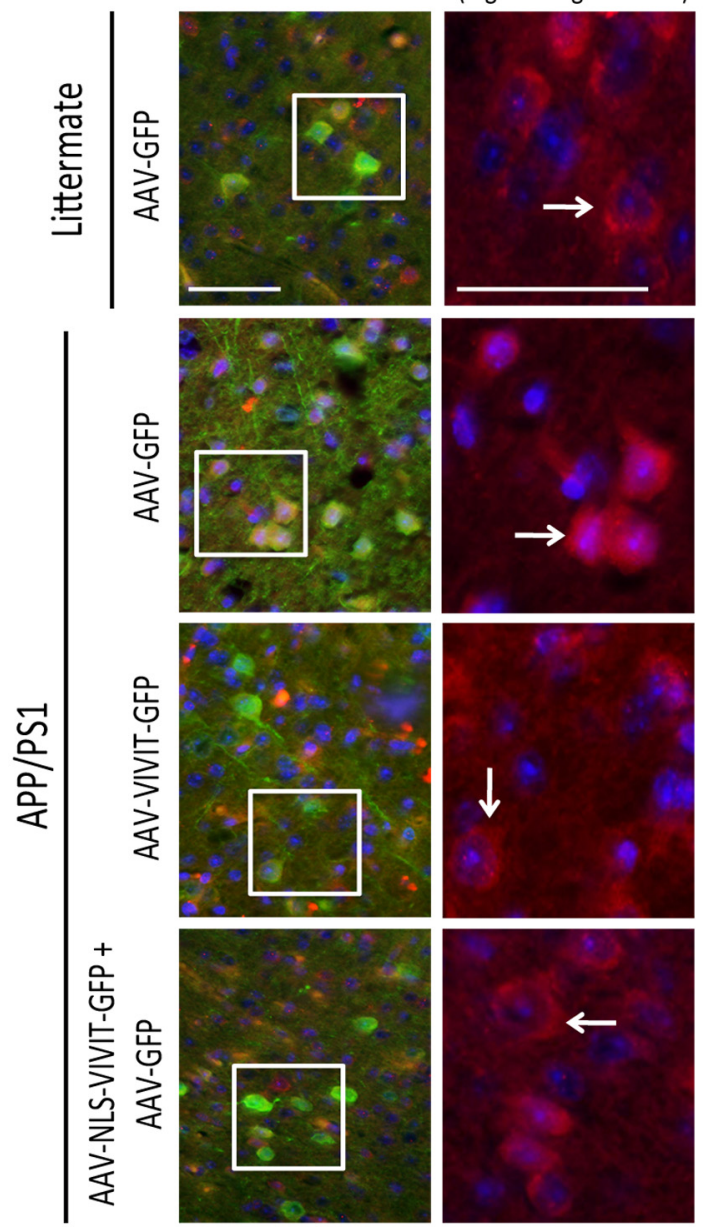

\section{B}

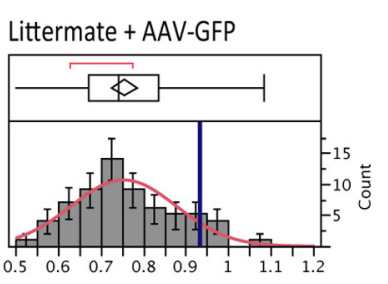

APP/PS1 + AAV-GFP

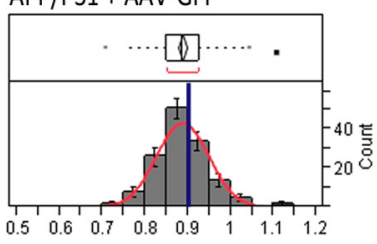

APP/PS1 + AAV-VIVIT-GFP

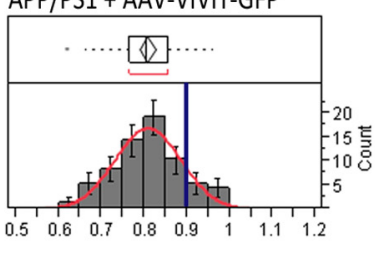

APP/PS1 + AAV-NLS-VIVIT-GFP

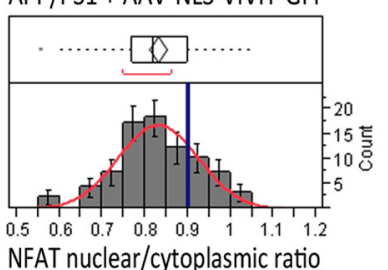

Figure 10. Overexpression of VIVIT-GFP and NLS-VIVIT-GFP prevent NFATC4 accumulation in the nucleus of transduced neurons. $A$, Representative images of GFP immunostaining and endogenous NFATc4 in injected animals allows detection of endogenous NFATc4 in transduced neurons. Scale bar, $50 \mu \mathrm{m}$; for higher magnification images, on the right, $20 \mu \mathrm{m}$. Higher magnification images of NFAT 4 and DAPI (third column) show that an increased recruitment of NFATC4 is detected in AAV-GFP-injected APP/PS1 mice. $\boldsymbol{B}$, The ratios of NFATC4 immunofluorescence intensity between the nucleus and the cytoplasm follow a normal distribution in all three groups tested. However, there is a shift toward higher values in AAV-GFP-injected APP/PS1 mice compared with AAV-VIVIT and AAV-NLS-VIVIT treated animals. On the graph, the blue line represents the mean of NFATc4 nuclear/cytoplasmic ratio in AAV-GFP injected APP/PS1 mice. The nuclear/cytoplasmic ratio is significantly increased in AAV-GFP treated transgenic mice compared with AAV-VIVIT-GFP and AAV-NLS-VIVIT-GFP $(p<0.05)$. ( $n<4$ animals per group and 3 slices per animal were analyzed).

tively active form of the transcription factor NFAT is introduced. The results are consistent with the hypothesis that the neurotoxic effects of $\mathrm{A} \beta$ are mediated, at least in part, by the transcriptional activation of NFAT downstream target genes. Although the contribution of NFAT to neurological function or dysfunction has received little attention, recent findings have demonstrated a role of NFAT in synaptic plasticity during development (Nguyen and Di Giovanni, 2008; Schwartz et al., 2009). Moreover, NFAT shows an abnormal nuclear accumulation in the brains of $\mathrm{AD}$ patients (Wu et al., 2010), which directly correlates with the levels of soluble $A \beta_{(1-42)}$ and the severity of cognitive impairment (Abdul et al., 2009). Our study provides evidence supporting the idea that NFAT is a specific molecular mediator of transcription-dependent modifications of the CNS structure in the context of Alzheimer's disease.

In vitro, we observed that the morphological changes induced by constitutively active calcineurin or by constitutively active

NFAT replicate the decrease of spine density and dendritic simplification induced by the neurotoxic $A \beta$ peptides. Along with recent data indicating that calcineurin is activated both in human $\mathrm{AD}$ brain and in transgenic models of $\mathrm{AD}$ (Liu et al., 2005; Dineley et al., 2007; Wu et al., 2010), we tested the hypothesis that inhibition of the excessive NFAT activity might improve the neuritic abnormalities observed in $\mathrm{AD}$ models. We demonstrate that VIVIT, a genetically encoded inhibitor of the interaction between calcineurin and NFAT, increases both the spine density and the complexity of dendritic arbors in $\mathrm{APP}^{\text {swe }}$-transgenic neurons or in $\mathrm{TgCM}$ treated wild-type neurons. In vivo, a gene transfer approach using adeno-associated vectors enabled the delivery of VIVIT to neurons and significantly increased the spine density in the vicinity of senile plaques. Transduction with an inhibitor that, due to a nuclear localization signal (NLS-VIVIT), specifically blocked the nuclear activation of NFAT, was also able to restore to nearly normal the morphological abnormalities associated with $\mathrm{A} \beta$. The ability of NLS-VIVIT to reproduce the same beneficial effects as VIVIT is intriguing, as this peptide is thought to interact primarily with the docking site of NFAT upon CaN. However, several studies demonstrated that activated $\mathrm{CaN}$ is able to translocate to the nucleus in neurons (Pujol et al., 1993; Solà et al., 1999; Yang et al., 2005; Schwartz et al., 2009), a phenomenon we also previously described in AD patients (Wu et al., 2010). We therefore propose that both $\mathrm{CaN}$ and NFAT translocate to the nuclear compartment, where VIVIT would compete with NFAT for CaN binding. Considering the higher affinity of VIVIT for CaN [ $\sim 25$-fold compared with NFAT (Aramburu et al., 1999)], the CaN/NFAT interaction would thus be interrupted and NFAT would rapidly be phosphorylated and shuttled back to the cytoplasm. This hypothesis is in agreement with the previous observation that NLS-VIVIT efficiently inhibited the expression of NFAT-target genes (Schwartz et al., 2009), and with our observation of a significant decrease in the NFAT nuclear/cytoplasmic ratio not only in AAV-VIVIT-GFP-injected animals but also in AAV-NLSVIVIT-treated mice.

Our results indicate that (1) NFAT activation is a likely consequence of $A \beta$ accumulation in Alzheimer's disease, as suggested by its prominent nuclear localization in the brain of $\mathrm{AD}$ patients (Abdul et al., 2009; Wu et al., 2010), and by the ability of VIVIT to restore $A \beta$-associated morphological neurodegenerative changes; (2) once activated and translocated to the nucleus, NFAT presumably induces the transcription of target genes in mature neurons that lead to a pathological remodeling of dendrites and dendritic spines. The beneficial effect of a nucleusdirected NFAT inhibitor supports the role of transcriptional events in the regulation of dendritic spine stability in vitro and in 
vivo. To some extent, these findings are unexpected, as the modulation of spine morphology has been largely focused on endocytosis and stability of AMPAR and NMDAR receptors, rather than transcriptionally regulated phenomena (Lau and Zukin, 2007; Zhang et al., 2008; Bhattacharyya et al., 2009; GoebelGoody et al., 2009). Although local spine-specific phenomena will ultimately determine the stability of individual spines, the current data emphasize that dendritic trees and spine density are also impacted at a transcriptional level. This conclusion is in accordance with a growing literature suggesting that transcriptional modulation can affect memory function (Saura and Valero, 2011), and with recent data demonstrating that alterations in mRNA trafficking and stability can lead to neural system alterations and degeneration in disorders as disparate as autism and amyotrophic lateral sclerosis (Gatto and Broadie, 2010).

The literature addressing the question of NFAT target genes specifically regulated in neurons is quite limited, but few interesting findings might be relevant in the context of Alzheimer's disease. For example, NFAT was shown to contribute to the induction of apoptosis in neuroblastoma cells through upregulation of Fas Ligand (Fas-L) (Luoma and Zirpel, 2008; Alvarez et al., 2011), a gene that was also reported to increase in AD brains, especially in the hippocampal formation and entorhinal cortex that are primarily involved in memory encoding. In addition, $\mathrm{A} \beta$ peptides induce a pathological increase in Fas-L in cortical primary neurons and its accumulation was demonstrated in neuritic dystrophies surrounding the amyloid deposits (Su et al., 2003). Even though no direct connection between Fas-L and spine morphology has been reported so far, one of the down-stream target of Fas-L, capsase-3, was recently shown to trigger early synaptic dysfunction and spine loss in AD (D'Amelio et al., 2011). This would suggest that activation of caspase- 3 may not only be involved in cell death but is also closely associated with the regulation of synaptic plasticity. It is therefore possible that the activation of Fas-L expression by NFAT indirectly causes a synaptic failure via the induction of a non-apoptotic caspase- 3 pathway. The expression of the potassium channel Kv2.1 can also be driven by NFAT and was shown to be upregulated by $72 \%$ in the hippocampus of rat injected with $\mathrm{A} \beta_{25-35}$. (Pan et al., 2004). Interestingly, the use of several potassium channel openers (minoxidil, pinacidil, cromakalim) can induce amnesia in mice (Ghelardini et al., 1998), thus suggesting that the regulation of the activity of the potassium channels might impair memory encoding, one of the best hallmark of $\mathrm{AD}$. As learning and memory deficits mainly reflect a default in spine-mediated plasticity, we can hypothesize that dysregulation of the potassium channel Kv2.1 might lead to the alteration of dendritic spine morphology. Last, the upregulation of the inositol 1,4,5-trisphosphate type 1 receptor (InsP3R) gene by NFAT is of particular interest in the context of Alzheimer's disease (Genazzani et al., 1999; Amberg et al., 2004). Indeed, InsP3R was shown to directly interact with presenilins 1 and 2, increasing its activity in response to Inositol3 -phosphate, thus leading to an excessive release of $\mathrm{Ca}^{2+}$ (Müller et al., 2011). The upregulation of InsP3R might therefore participate to exacerbate the increase of the resting calcium levels. The few examples mentioned above might be of interest in the context of $\mathrm{AD}$, but other NFAT target genes previously identified does not seem relevant in a pathological context. Indeed, NFAT transcriptional activity was associated with an increased expression of the neurotrophic factor BDNF in Purkinje cells, an important mediator of axonal outgrowth during development (Graef et al., 2003). These varied results could be explained by the ability of NFAT to interact with other transcriptional factors (Crabtree and
Olson, 2002; Ogata et al., 2003; Kao et al., 2009), a mechanism by which the pattern of NFAT-induced target genes can be modulated depending on the developmental state, on the cell type, and on the physiological or pathological context. It is therefore conceivable that a particular set of NFAT coactivators are present in cortical neurons exposed to $\mathrm{A} \beta$, so that its activation leads to the upregulation of genes implicated in dendritic spine shrinkage.

Although the transcriptional effects of NFAT (and its inhibition) await further investigation, the present study provides a novel neuroprotective therapeutic target against the downstream neurotoxic effects of amyloid- $\beta$ and confirms the feasibility to pharmacologically arrest the "amyloid cascade" of neurodegeneration at a step after amyloid deposition has already occurred. The latter has critical therapeutic implications, since most amyloid deposition is thought to occur years or even decades before the onset of cognitive decline, and therefore, before AD diagnosis, whereas synaptic and neuron loss largely occurs in a second stage and correlates with the severity of cognitive impairment (Sperling et al., 2009). In this scenario, amyloid-directed therapies would not be sufficiently effective once cognitive symptoms (and the underlying neurodegenerative processes) have begun. Consequently, combined synergistic therapies aimed at reducing $A \beta$ content (either by inhibiting the production of $A \beta$ peptides or by increasing their clearance) and at restoring $\mathrm{A} \beta$-induced neural damage would be needed.

\section{References}

Abdul HM, Sama MA, Furman JL, Mathis DM, Beckett TL, Weidner AM, Patel ES, Baig I, Murphy MP, LeVine H 3rd, Kraner SD, Norris CM (2009) Cognitive decline in Alzheimer's disease is associated with selective changes in calcineurin/NFAT signaling. J Neurosci 29:12957-12969.

Alvarez S, Blanco A, Fresno M, Muñoz-Fernández MÁ (2011) TNF-alpha contributes to caspase-3 independent apoptosis in neuroblastoma cells: role of NFAT. PLoS One 6:e16100.

Amberg GC, Rossow CF, Navedo MF, Santana LF (2004) NFATc3 regulates Kv2.1 expression in arterial smooth muscle. J Biol Chem 279:47326-47334.

Aramburu J, Yaffe MB, López-Rodríguez C, Cantley LC, Hogan PG, Rao A (1999) Affinity-driven peptide selection of an NFAT inhibitor more selective than cyclosporin A. Science 285:2129-2133.

Bacskai BJ, Klunk WE, Mathis CA, Hyman BT (2002) Imaging amyloidbeta deposits in vivo. J Cereb Blood Flow Metab 22:1035-1041.

Benedito AB, Lehtinen M, Massol R, Lopes UG, Kirchhausen T, Rao A, Bonni A (2005) The transcription factor NFAT3 mediates neuronal survival. J Biol Chem 280:2818-2825.

Bhattacharyya S, Biou V, Xu W, Schlüter O, Malenka RC (2009) A critical role for PSD-95/AKAP interactions in endocytosis of synaptic AMPA receptors. Nat Neurosci 12:172-181.

Busche MA, Eichhoff G, Adelsberger H, Abramowski D, Wiederhold KH, Haass C, Staufenbiel M, Konnerth A, Garaschuk O (2008) Clusters of hyperactive neurons near amyloid plaques in a mouse model of Alzheimer's disease. Science 321:1686-1689.

Crabtree GR, Olson EN (2002) NFAT signaling: choreographing the social lives of cells. Cell 109 [Suppl]:S67-S79.

D’Amelio M, Cavallucci V, Middei S, Marchetti C, Pacioni S, Ferri A, Diamantini A, De Zio D, Carrara P, Battistini L, Moreno S, Bacci A, Ammassari-Teule M, Marie H, Cecconi F (2011) Caspase-3 triggers early synaptic dysfunction in a mouse model of Alzheimer's disease. Nat Neurosci 14:69-76.

DeKosky ST, Scheff SW, Styren SD (1996) Structural correlates of cognition in dementia: quantification and assessment of synapse change. Neurodegeneration 5:417-421.

Dell'Acqua ML, Dodge KL, Tavalin SJ, Scott JD (2002) Mapping the protein phosphatase-2B anchoring site on AKAP79. Binding and inhibition of phosphatase activity are mediated by residues 315-360. J Biol Chem 277:48796-48802.

Dineley KT, Hogan D, Zhang WR, Taglialatela G (2007) Acute inhibition of calcineurin restores associative learning and memory in $\operatorname{Tg} 2576$ APP transgenic mice. Neurobiol Learn Mem 88:217-224. 
Gatto CL, Broadie K (2010) Genetic controls balancing excitatory and inhibitory synaptogenesis in neurodevelopmental disorder models. Front Synaptic Neurosci 2:4.

Genazzani AA, Carafoli E, Guerini D (1999) Calcineurin controls inositol 1,4,5-trisphosphate type 1 receptor expression in neurons. Proc Natl Acad Sci U S A 96:5797-5801.

Ghelardini C, Galeotti N, Bartolini A (1998) Influence of potassium channel modulators on cognitive processes in mice. Br J Pharmacol 123:1079-1084.

Goebel-Goody SM, Davies KD, Alvestad Linger RM, Freund RK, Browning MD (2009) Phospho-regulation of synaptic and extrasynaptic $\mathrm{N}$-methyl-d-aspartate receptors in adult hippocampal slices. Neuroscience 158:1446-1459.

Graef IA, Wang F, Charron F, Chen L, Neilson J, Tessier-Lavigne M, Crabtree GR (2003) Neurotrophins and netrins require calcineurin/NFAT signaling to stimulate outgrowth of embryonic axons. Cell 113:657-670.

Groth RD, Dunbar RL, Mermelstein PG (2003) Calcineurin regulation of neuronal plasticity. Biochem Biophys Res Commun 311:1159-1171.

Ho AM, Jain J, Rao A, Hogan PG (1994) Expression of the transcription factor NFATp in a neuronal cell line and in the murine nervous system. J Biol Chem 269:28181-28186.

Holtmaat AJ, Trachtenberg JT, Wilbrecht L, Shepherd GM, Zhang X, Knott GW, Svoboda K (2005) Transient and persistent dendritic spines in the neocortex in vivo. Neuron 45:279-291.

Ingelsson M, Fukumoto $\mathrm{H}$, Newell KL, Growdon JH, Hedley-Whyte ET, Frosch MP, Albert MS, Hyman BT, Irizarry MC (2004) Early Abeta accumulation and progressive synaptic loss, gliosis, and tangle formation in AD brain. Neurology 62:925-931.

Jankowsky JL, Fadale DJ, Anderson J, Xu GM, Gonzales V, Jenkins NA, Copeland NG, Lee MK, Younkin LH, Wagner SL, Younkin SG, Borchelt DR (2004) Mutant presenilins specifically elevate the levels of the 42 residue beta-amyloid peptide in vivo: evidence for augmentation of a 42 -specific gamma secretase. Hum Mol Genet 13:159-170.

Jurado S, Biou V, Malenka RC (2010) A calcineurin/AKAP complex is required for NMDA receptor-dependent long-term depression. Nat Neurosci 13:1053-1055.

Kao SC, Wu H, Xie J, Chang CP, Ranish JA, Graef IA, Crabtree GR (2009) Calcineurin/NFAT signaling is required for neuregulin-regulated Schwann cell differentiation. Science 323:651-654.

Klee CB, Crouch TH, Krinks MH (1979) Calcineurin: a calcium- and calmodulin-binding protein of the nervous system. Proc Natl Acad Sci U S A 76:6270-6273.

Klunk WE, Bacskai BJ, Mathis CA, Kajdasz ST, McLellan ME, Frosch MP, Debnath ML, Holt DP, Wang Y, Hyman BT (2002) Imaging Abeta plaques in living transgenic mice with multiphoton microscopy and methoxy-X04, a systemically administered Congo red derivative. J Neuropathol Exp Neurol 61:797-805.

Knowles RB, Wyart C, Buldyrev SV, Cruz L, Urbanc B, Hasselmo ME, Stanley HE, Hyman BT (1999) Plaque-induced neurite abnormalities: implications for disruption of neural networks in Alzheimer's disease. Proc Natl Acad Sci U S A 96:5274-5279.

Koffie RM, Meyer-Luehmann M, Hashimoto T, Adams KW, Mielke ML, Garcia-Alloza M, Micheva KD, Smith SJ, Kim ML, Lee VM, Hyman BT, Spires-Jones TL (2009) Oligomeric amyloid beta associates with postsynaptic densities and correlates with excitatory synapse loss near senile plaques. Proc Natl Acad Sci U S A 106:4012-4017.

Kuchibhotla KV, Goldman ST, Lattarulo CR, Wu HY, Hyman BT, Bacskai BJ (2008) Abeta plaques lead to aberrant regulation of calcium homeostasis in vivo resulting in structural and functional disruption of neuronal networks. Neuron 59:214-225.

Lau CG, Zukin RS (2007) NMDA receptor trafficking in synaptic plasticity and neuropsychiatric disorders. Nat Rev Neurosci 8:413-426.

Lee SH, Kim BC, Yang DH, Park MS, Choi SM, Kim MK, Cho KH (2008) Calcineurin inhibitor-mediated bilateral hippocampal injury after bone marrow transplantation. J Neurol 255:929-931.

Lesn é S, Koh MT, Kotilinek L, Kayed R, Glabe CG, Yang A, Gallagher M, Ashe $\mathrm{KH}$ (2006) A specific amyloid-beta protein assembly in the brain impairs memory. Nature 440:352-357.

Liu F, Grundke-Iqbal I, Iqbal K, Oda Y, Tomizawa K, Gong CX (2005) Truncation and activation of calcineurin A by calpain I in Alzheimer disease brain. J Biol Chem 280:37755-37762.
Liu JO (2003) Endogenous protein inhibitors of calcineurin. Biochem Biophys Res Commun 311:1103-1109.

Luoma JI, Zirpel L (2008) Deafferentation-induced activation of NFAT (nuclear factor of activated T-cells) in cochlear nucleus neurons during a developmental critical period: a role for NFATc4-dependent apoptosis in the CNS. J Neurosci 28:3159-3169.

Molkentin JD, Lu JR, Antos CL, Markham B, Richardson J, Robbins J, Grant SR, Olson EN (1998) A calcineurin-dependent transcriptional pathway for cardiac hypertrophy. Cell 93:215-228.

Müller M, Cheung KH, Foskett JK (2011) Enhanced ROS generation mediated by Alzheimer's disease presenilin regulation of InsP3R Ca ${ }^{2+}$ signaling. Antioxid Redox Signal 14:1225-1235.

Nguyen T, Di Giovanni S (2008) NFAT signaling in neural development and axon growth. Int J Dev Neurosci 26:141-145.

Norris CM, Kadish I, Blalock EM, Chen KC, Thibault V, Porter NM, Landfield PW, Kraner SD (2005) Calcineurin triggers reactive/inflammatory processes in astrocytes and is upregulated in aging and Alzheimer's models. J Neurosci 25:4649-4658.

Ogata K, Sato K, Tahirov TH (2003) Eukaryotic transcriptional regulatory complexes: cooperativity from near and afar. Curr Opin Struct Biol 13:40-48.

Pan Y, Xu X, Tong X, Wang X (2004) Messenger RNA and protein expression analysis of voltage-gated potassium channels in the brain of Abeta(25-35)-treated rats. J Neurosci Res 77:94-99.

Ponticelli C, Campise MR (2005) Neurological complications in kidney transplant recipients. J Nephrol 18:521-528.

Pujol MJ, Bosser R, Vendrell M, Serratosa J, Bachs O (1993) Nuclear calmodulin-binding proteins in rat neurons. J Neurochem 60:1422-1428.

Rodriguez A, Ehlenberger DB, Dickstein DL, Hof PR, Wearne SL (2008) Automated three-dimensional detection and shape classification of dendritic spines from fluorescence microscopy images. PLoS One 3:e1997.

Rozkalne A, Hyman BT, Spires-Jones TL (2011) Calcineurin inhibition with FK506 ameliorates dendritic spine density deficits in plaque-bearing Alzheimer model mice. Neurobiol Dis 41:650-654.

Saura CA, Valero J (2011) The role of CREB signaling in Alzheimer's disease and other cognitive disorders. Rev Neurosci 22:153-169.

Schwartz N, Schohl A, Ruthazer ES (2009) Neural activity regulates synaptic properties and dendritic structure in vivo through calcineurin/NFAT signaling. Neuron 62:655-669.

Shankar GM, Bloodgood BL, Townsend M, Walsh DM, Selkoe DJ, Sabatini BL (2007) Natural oligomers of the Alzheimer amyloid-beta protein induce reversible synapse loss by modulating an NMDA-type glutamate receptor-dependent signaling pathway. J Neurosci 27:2866-2875.

Shankar GM, Li S, Mehta TH, Garcia-Munoz A, Shepardson NE, Smith I, Brett FM, Farrell MA, Rowan MJ, Lemere CA, Regan CM, Walsh DM, Sabatini BL, Selkoe DJ (2008) Amyloid-beta protein dimers isolated directly from Alzheimer's brains impair synaptic plasticity and memory. Nat Med 14:837-842.

Sol à C, Tusell JM, Serratosa J (1999) Comparative study of the distribution of calmodulin kinase II and calcineurin in the mouse brain. J Neurosci Res 57:651-662.

Sperling RA, Laviolette PS, O'Keefe K, O’Brien J, Rentz DM, Pihlajamaki M, Marshall G, Hyman BT, Selkoe DJ, Hedden T, Buckner RL, Becker JA, Johnson KA (2009) Amyloid deposition is associated with impaired default network function in older persons without dementia. Neuron 63:178-188.

Spires TL, Meyer-Luehmann M, Stern EA, McLean PJ, Skoch J, Nguyen PT, Bacskai BJ, Hyman BT (2005) Dendritic spine abnormalities in amyloid precursor protein transgenic mice demonstrated by gene transfer and intravital multiphoton microscopy. J Neurosci 25:7278-7287.

Spires-Jones TL, Kay K, Matsouka R, Rozkalne A, Betensky RA, Hyman BT (2011) Calcineurin inhibition with systemic FK506 treatment increases dendritic branching and dendritic spine density in healthy adult mouse brain. Neurosci Lett 487:260-263.

Su JH, Anderson AJ, Cribbs DH, Tu C, Tong L, Kesslack P, Cotman CW (2003) Fas and Fas ligand are associated with neuritic degeneration in the $\mathrm{AD}$ brain and participate in beta-amyloid-induced neuronal death. Neurobiol Dis 12:182-193.

Taglialatela G, Hogan D, Zhang WR, Dineley KT (2009) Intermediate- and long-term recognition memory deficits in $\mathrm{Tg} 2576$ mice are reversed with acute calcineurin inhibition. Behav Brain Res 200:95-99. 
Terry RD, Masliah E, Salmon DP, Butters N, DeTeresa R, Hill R, Hansen LA, Katzman R (1991) Physical basis of cognitive alterations in Alzheimer's disease: synapse loss is the major correlate of cognitive impairment. Ann Neurol 30:572-580.

Walsh DM, Klyubin I, Fadeeva JV, Cullen WK, Anwyl R, Wolfe MS, Rowan MJ, Selkoe DJ (2002) Naturally secreted oligomers of amyloid beta protein potently inhibit hippocampal long-term potentiation in vivo. Nature 416:535-539.

Wu HY, Tomizawa K, Oda Y, Wei FY, Lu YF, Matsushita M, Li ST, Moriwaki A, Matsui H (2004) Critical role of calpain-mediated cleavage of calcineurin in excitotoxic neurodegeneration. J Biol Chem 279:4929-4940.

Wu HY, Hudry E, Hashimoto T, Kuchibhotla K, Rozkalne A, Fan Z, SpiresJones T, Xie H, Arbel-Ornath M, Grosskreutz CL, Bacskai BJ, Hyman BT
(2010) Amyloid beta induces the morphological neurodegenerative triad of spine loss, dendritic simplification, and neuritic dystrophies through calcineurin activation. J Neurosci 30:2636-2649.

Yang Y, Fischer QS, Zhang Y, Baumgärtel K, Mansuy IM, Daw NW (2005) Reversible blockade of experience-dependent plasticity by calcineurin in mouse visual cortex. Nat Neurosci 8:791-796.

Zhang Y, Venkitaramani DV, Gladding CM, Kurup P, Molnar E, Collingridge GL, Lombroso PJ (2008) The tyrosine phosphatase STEP mediates AMPA receptor endocytosis after metabotropic glutamate receptor stimulation. J Neurosci 28:10561-10566.

Zhou Q, Homma KJ, Poo MM (2004) Shrinkage of dendritic spines associated with long-term depression of hippocampal synapses. Neuron 44: 749-757. 